\title{
A Comprehensive Overview of TCP Congestion Control in 5G Networks: Research Challenges and Future Perspectives
}

\author{
Josip Lorincz $^{1, * \mathbb{C}}$, Zvonimir Klarin ${ }^{2} \mathbb{D}$ and Julije Ožegović ${ }^{1}$ \\ 1 Faculty of Electrical Engineering, Mechanical Engineering and Naval Architecture (FESB), University of Split, \\ 21000 Split, Croatia; julije.ozegovic@fesb.hr \\ 2 Polytechnic of Sibenik, Trg Andrije Hebranga 11, 22000 Sibenik, Croatia; zklarin@vus.hr \\ * Correspondence: josip.lorincz@fesb.hr
}

Citation: Lorincz, J.; Klarin, Z.; Ožegović, J. A Comprehensive Overview of TCP Congestion Control in 5G Networks: Research Challenges and Future Perspectives. Sensors 2021, 21, 4510. https://doi.org/10.3390/ s21134510

Academic Editor: Gianluca Reali

Received: 31 May 2021

Accepted: 28 June 2021

Published: 30 June 2021

Publisher's Note: MDPI stays neutral with regard to jurisdictional claims in published maps and institutional affiliations.

Copyright: (c) 2021 by the authors. Licensee MDPI, Basel, Switzerland. This article is an open access article distributed under the terms and conditions of the Creative Commons Attribution (CC BY) license (https:// creativecommons.org/licenses/by/ $4.0 /)$.
Abstract: In today's data networks, the main protocol used to ensure reliable communications is the transmission control protocol (TCP). The TCP performance is largely determined by the used congestion control (CC) algorithm. TCP CC algorithms have evolved over the past three decades and a large number of $\mathrm{CC}$ algorithm variations have been developed to accommodate various network environments. The fifth-generation (5G) mobile network presents a new challenge for the implementation of the TCP CC mechanism, since networks will operate in environments with huge user device density and vast traffic flows. In contrast to the pre-5G networks that operate in the sub- $6 \mathrm{GHz}$ bands, the implementation of TCP CC algorithms in 5G mmWave communications will be further compromised with high variations in channel quality and susceptibility to blockages due to high penetration losses and atmospheric absorptions. These challenges will be particularly present in environments such as sensor networks and Internet of Things (IoT) applications. To alleviate these challenges, this paper provides an overview of the most popular single-flow and multy-flow TCP CC algorithms used in pre-5G networks. The related work on the previous examinations of TCP CC algorithm performance in $5 \mathrm{G}$ networks is further presented. A possible implementation of TCP CC algorithms is thoroughly analysed with respect to the specificities of $5 \mathrm{G}$ networks, such as the usage of high frequencies in the mmWave spectrum, the frequent horizontal and vertical handovers, the implementation of the 5G core network, the usage of beamforming and data buffering, the exploitation of edge computing, and the constantly transmitted always-on signals. Moreover, the capabilities of machine learning technique implementations for the improvement of TCPs CC performance have been presented last, with a discussion on future research opportunities that can contribute to the improvement of TCP CC implementation in 5G networks. This survey paper can serve as the basis for the development of novel solutions that will ensure the reliable implementation of TCP CC in different usage scenarios of $5 \mathrm{G}$ networks.

Keywords: TCP; congestion control; 5G; mmWave; network; mobile; algorithms; communications; wireless

\section{Introduction}

For every new generation of mobile network, the demand for new services and use cases increases. Today's trends of ubiquitous computing tend to incorporate technology into every device, changing the way that we perform our daily activities and do business. Accommodating these trends imposes implementation and operation challenges for mobile network operators. To satisfy future needs, the International Telecommunications UnionRadiocommunications Sector (ITU-R) has defined three main technology advancements that fifth-generation (5G) mobile networks should support (Figure 1) [1]: ultra-reliable and low latency communications (URLLC), enhanced mobile broadband (eMBB), and massive machine-type communications (mMTC) in sensor networks. 


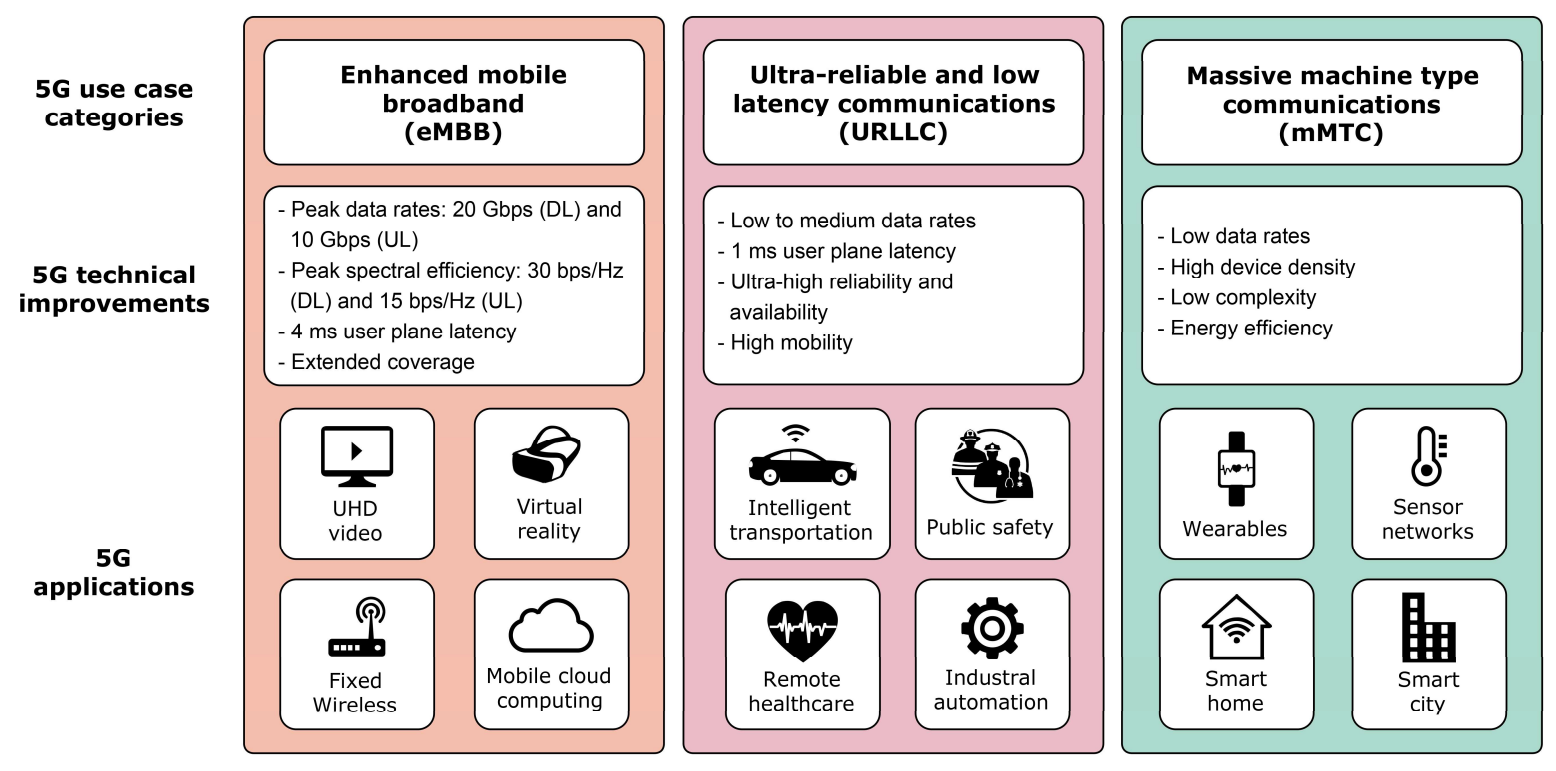

Figure 1. Three main use cases of $5 \mathrm{G}$ mobile networks.

The usage scenarios characterised as eMBB aim to provide high data rates, high traffic capacity, and high seamless mobility for both hotspots and wide-area coverage [1]. As 5G mobile technology is implemented in phases, its full potential being reached in the upcoming years will enable eMBB use cases to benefit the majority of the population in this early phase of $5 \mathrm{G}$ mobile network development (Figure 1). The reason for this is that the initial implementation of 5G networks is aligned with the consumer market needs, supporting a rising number of smartphone subscriptions accompanied by an increasing mobile data growth rate [2]. In Ref. [3], the minimum requirements for the peak data rate in the eMBB usage scenario are defined as $20 \mathrm{Gbit} / \mathrm{s}$ for downlink, $10 \mathrm{Gbit} / \mathrm{s}$ for uplink, and $4 \mathrm{~ms}$ for user plane latency. These performance targets are assumed for scenarios with a single user transferring small internet protocol (IP) packets for both the downlink and uplink.

The URLLC use case presents applications that require ultra-low latencies, reliable communication and high availability (Figure 1). High reliability and low latency are crucial requirements for emerging mission-critical applications including telesurgery, intelligent transportation and industrial automation [4]. In URLLC usage scenarios, the minimum requirement for user plane latency for small IP packets is $1 \mathrm{~ms}$ for both downlink and uplink transmission [3].

Machine-to-machine communication (M2M) represents machine-centric communication between different devices and sensors without human interaction (Figure 1). With the increasing number of devices and sensors offering M2M communication, mMTC use cases will become more present in the near future. The main driver of increasing machine-type communication is the advent of sensor networks as part of the Internet of Things (IoT) paradigm. This is where a very large number of devices and sensors will communicate and exchange information. These devices and sensors are characterised as low cost and low power with a very long battery life. These devices and sensors are usually transmitting a small amount of data that is not sensitive to delays [1].

Previous generations of cellular networks e.g., 3rd (3G) and 4th (4G), have used frequency bands under $6 \mathrm{GHz}$. As these frequencies are becoming increasingly saturated and as there are limitations that mean that they cannot fulfil the new increasing demands, the need for higher frequency bands above $6 \mathrm{GHz}$ has arisen. Accordingly, 5G mobile networks are designed to operate on a variety of frequency bands, including the sub- $6 \mathrm{GHz}$ band used by the previous generations of mobile networks and the above $6 \mathrm{GHz}$ frequency bands as well. 
This is why $5 \mathrm{G}$ technology is also known as 5G New Radio (NR). For the first time in the history of mobile network generations, information exchange uses a frequency spectrum known as millimetre-wave (mmWave) characterized with signal transmission at carrier frequencies larger than $24 \mathrm{GHz}$. The main reason for using these frequencies is to accommodate the emerging high requirements for the larger data rates [5]. Within the mmWave frequency range, up to $100 \mathrm{GHz}$ of a possible new spectrum is expected to be available for cellular mobile communications [6]. In contrast to the previously available spectrum in the sub- $6 \mathrm{GHz}$ band, this represents a significant spectrum increase aimed for usage in $5 \mathrm{G}$ networks.

Although mmWave frequency bands classified by the 3rd generation partnership project (3GPP) as FR2 are expected to bring major throughput improvements in mobile communications, sub-6 GHz frequencies classified by 3GPP as FR1 frequency band will still be predominantly used in the initial launches of 5G networks. To accommodate specific purposes, the $5 \mathrm{G}$ frequency spectrum is divided into three broad categories (Figure 2): low-bands (sub-1GHz), mid-bands (1-6 GHz), and high-bands (24-52 GHz) [7]. Lowbands are required for wide-area coverage, signal coverage inside buildings and to support IoT use cases. Low-band frequencies of $600-900 \mathrm{MHz}$ are mainly considered across the world [8]. The mid-band spectrum offers a trade-off between coverage and capacity. Most of the commercial $5 \mathrm{G}$ networks will use $3.3 \mathrm{GHz}$ to $4.2 \mathrm{GHz}$ range in the mid-band spectrum [8]. High-band frequencies are required to achieve ultra-high data rates and ultra-low latencies that are expected in 5G communication. Allocated global mmWave spectrum includes $24-28 \mathrm{GHz}$ frequencies, with more spectrum allocation planned in the future (e.g., 37-50 GHz, 50-71 GHz) [8].

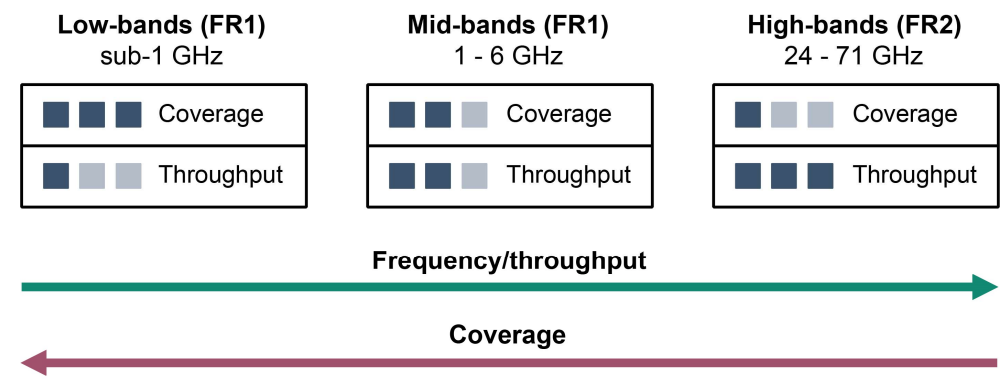

Figure 2. Frequency bands in the 5G networks.

The main advantage of deploying mmWave frequencies is the ability to deliver high data rates using the large bandwidth. Due to the short wavelengths (below $10 \mathrm{~mm}$ ) of the high-frequency waves, transmitting and receiving antennas can be produced in compact sizes and easily implemented within base stations (BSs) and user equipment (UE). Ultra-dense antenna arrays deployed within a BS can produce narrow directional beams, and therefore enable frequency reuse among concurrent transmissions within a small geographic area [9].

However, the propagation attributes of mmWave frequencies have some drawbacks that $5 \mathrm{G}$ mobile networks need to overcome. The main drawbacks that are facing mmWave communication systems are increased path loss, susceptibility to blockages and atmospheric absorption [10]. The use of high frequencies implies that the transmitted signals have short wavelengths, which are very sensitive to the blockages caused by various obstacles [11]. Additionally, user mobility causes shifts in the mmWave communication from both line-of-sight (LOS) and non-line-of-sight (NLOS) transmissions. This results in severe signal degradation due to the various objects blocking the signal propagation.

Some approaches such as massive multiple-input and multiple-output (mMIMO) transmissions with beam-forming and small cell densification have been developed to address these shortcomings. However, a problem that arises using directional beamforming 
is beam misalignment. This problem is characterised with transmitter's beam not aligned with the receiver's beam, causing signal degradation or even a loss of connection.

Besides affecting the physical and media access control (MAC) layers, ensuring the high data rates in environments characterised with fluctuations in channel quality will also deteriorate the performance of the transport layer of the network [12]. Ensuring reliable endto-end connections over 5G mobile networks, especially when operating in the mmWave spectrum, presents a demanding challenge in terms of its practical implementation. This challenge arose during the last years of the subject of the researchers' interest [13]. In the transport layer, the transmission control protocol (TCP) is a major protocol used in today's communication over the Internet. The TCP is designed to provide reliable end-to-end connection over unreliable links. One of the mechanisms that plays a critical role in TCP performance is congestion control (CC).

One of the main purposes of the CC mechanism is to probe the network for available capacity by exponentially increasing the window size. This approach enables avoiding the congestion of the network that can be caused by a large burst of data at the beginning of transmission [14]. Blockage and misalignment problems can seriously affect the TCP CC mechanism, resulting in poor end-to-end transmission performance. Conventional TCP CC algorithms are not able to differentiate between the potential causes of packet loss. They can occur due to congestion or poor channel quality caused by the blockage or beam misalignment [15]. To achieve the specified performance and to accommodate the $5 \mathrm{G}$ requirements, the aforementioned shortcomings need to be addressed. In this paper, the TCP CC performance in $5 \mathrm{G} \mathrm{mmWave} \mathrm{communication} \mathrm{systems} \mathrm{has} \mathrm{been} \mathrm{analysed.} \mathrm{The}$ paper investigates the recent works regarding TCP CC in 5G networks and discusses the opportunities and future research challenges related to improving TPC CC in 5G networks operating in the sub- $6 \mathrm{GHz}$ and mmWave spectrum.

The rest of the paper is organised as follows. The TCP CC mechanism is explained and a brief review of the popular single and multipath CC algorithms is presented in Section 2. In Section 3, the investigation into the related works dedicated to the CC algorithms used in $5 \mathrm{G}$ networks is given. Section 4 overviews the future challenges in the realisation of TCP CC regarding the different technological advancements that will be implemented in $5 \mathrm{G}$ networks. In Section 5, an overview of the possible implementation of machine learning for improving TCP CC in 5G networks is presented. The research challenges and future directions that can contribute to enhancing TCP CC in 5G networks are discussed in Section 6. Finally, some concluding remarks are given in Section 7.

\section{TCP Congestion Control}

The TCP is a connection-oriented, reliable end-to-end protocol that guarantees the ordered delivery of byte streams in a full-duplex inter-process communication $[16,17]$. Initially, TCP was developed as a protocol to support military computer communication systems. Nowadays, TCP is de facto the standard for reliable end-to-end communication over the Internet. The TCP is responsible for establishing and terminating the connection, the reliable communication between hosts, the flow control, and the CC over unreliable networks.

As defined in Ref. [17], the process of establishing a TCP connection involves a threeway handshake (3WHS) procedure. Since the 3 WHS process increases the latency of TCP flows, especially short flows that are common in today's web services, a new mechanism called TCP fast open (TFO) has been proposed [18]. TFO is an optional mechanism that decreases latency by eliminating one full round-trip-time (RTT). This RTT elimination is performed by allowing data exchange before the standard 3WHS process is completed. This is achieved by enabling data packets to be transferred in SYN and SYN-ACK packets, and as such delivered to the application at receiving end [18]. TCP uses a flow control mechanism to determine how much data the receiver is able to accept. The receiver uses a window size field in the TCP header, which represents the available buffer size of the receiver. This informs the sender about the maximum number of bytes that they are 
allowed to transmit [19]. The purpose of the TCP CC mechanism is to avoid congestion of the network.

\subsection{Network Congestion and TCP CC Mechanism}

Network congestion is the result of a network node being overwhelmed with more data than it can process. Buffers are added to the communication nodes to prevent packet loss caused by the burst of packets. This consequently increases the delay of each packet passing through the buffer, which results in the degradation of the network performance [20]. On the other hand, network nodes with large buffers can cause an excess buffering of the packets. This is known as a bufferbloat problem [21]. This consequently leads to long queuing delays and overall network throughput degradation. The maximum bandwidth of the network link is referred to as a bottleneck bandwidth and it is determined by the bandwidth of the fully saturated network link. In a scenario where the TCP data rate is less than the bottleneck bandwidth, no congestion occurs and the delivery rate corresponds to the sending rate [20]. As the sending rate exceeds the bottleneck bandwidth, the buffers start to fill to the point where the buffers are full, causing the network nodes to start dropping packets. The optimal operation point of TCP is the point where the sending rate is equal to the bottleneck bandwidth. The TCP CC is a mechanism that aims to prevent network congestion and to ensure efficient network utilisation while working near to the optimal operation point.

The TCP CC is one of the main parts of the TCP protocol. Over the years, it has experienced numerous improvements through the application of different algorithms. The first TCP specification considered only flow control as a mechanism to prevent the buffer overflowing on the receiver end, while neglecting the congestion of the network itself [17]. The general idea of TCP CC is to prevent the sender from overflowing the network by determining the available capacity. Congestion avoidance and control were first introduced in 1988 [22] after a series of "congestion collapses" occurred on the Internet. The standard TCP CC mechanism is based on the additive increase multiplicative decrease (AIMD) algorithm, which incorporates four phases: slow start, congestion avoidance, fast retransmit and fast recovery [14]. An initial version of CC only requires the implementation of a slow start and the congestion avoidance phase. The fast retransmit and fast recovery phases were introduced later.

\subsubsection{The Slow Start and Congestion Avoidance TCP Mechanism}

The interdependence between the congestion window (cwnd) size and the transmission RTT of the TCP segment for the TCP version based on a slow start with congestion avoidance has been presented in Figure 3a. In this approach, the slow start and congestion avoidance phases are implemented using a stated cwnd variable that controls the amount of data that can be sent before receiving an ACK (Figure 3a). The cwnd size is a senderspecific variable that is maintained for each TCP session. The bandwidth-delay product (BDP), which determines the maximum amount of incoming data over a network link is considered to be the ideal value of the cwnd size. The network congestion is evaluated based on the received ACK packets of transmitted data. The slow start phase is the initial phase in the beginning of transmission (Figure 3a). In this phase, the TCP quickly probes the available capacity while avoiding the network congestion that can occur in the beginning of the transmission. After every successful transmission of data and the reception of an appropriate ACK for said data, cwnd is increased and the sender can send more data (Figure 3a). After the three-way handshake process, the initial window (iw) is determined and can be large as 10 maximum segment size (MSS) [23]. In the slow start phase, the cwnd is increased by one MSS for each ACK received, doubling its size for every RTT. The cwnd will continue increasing the rate until either the ssthresh (slow-start threshold) is reached (Figure 3a), packet loss occurs or cwnd exceeds the rwnd (receiver window) size. 


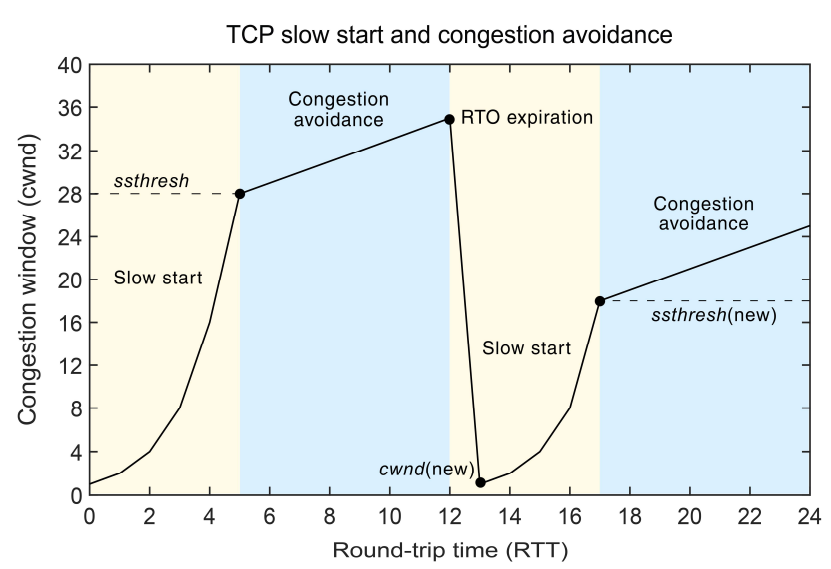

(a)

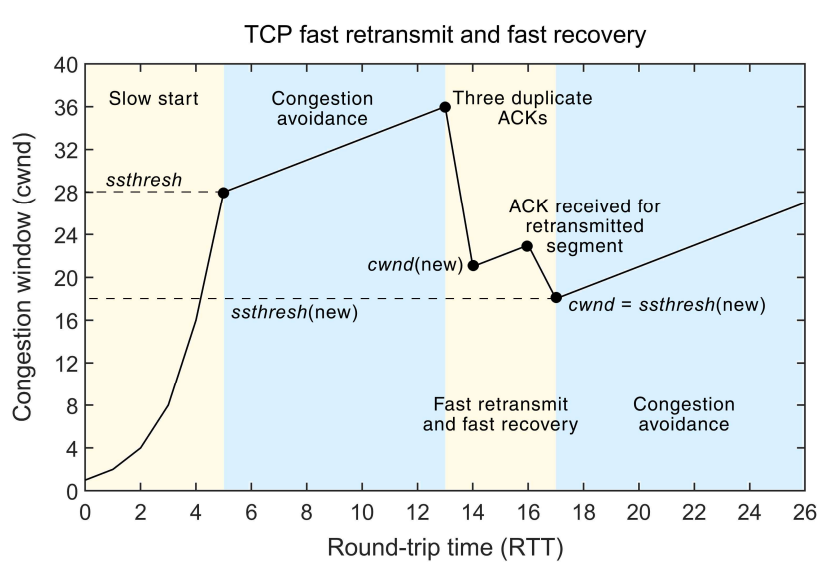

(b)

Figure 3. Standard working principles of the TCP CC mechanism related to the interdependence between cwnd and RTT for: (a) the TCP slow start and congestion avoidance mechanism; (b) the TCP fast retransmit and fast recovery mechanism.

After cwnd exceeds the ssthresh size, the TCP CC mechanism enters the congestion avoidance phase (Figure 3a). The purpose of the congestion avoidance phase is to slowly probe the network for more available capacity, by increasing the cwnd less aggressively than in the slow start phase. The connection stays in the congestion avoidance phase until congestion is detected. If congestion is detected during the congestion avoidance phase using the retransmission time-out (RTO) expiration parameter, the TCP connection enters the slow start phase where the ssthresh is set to half of the current cwnd size and the cwnd is decreased to one MSS (Figure 3a). The process of adjusting the congestion window (cwnd) continues according to the described mechanism.

\subsubsection{The Fast Retransmit and Fast Recovery TCP Mechanism}

Waiting for the RTO to expire leads to a long period of connection inactivity. Because of that, a new mechanism called fast retransmit with fast recovery was introduced as a part of the TCP CC [24] (Figure 3b). The interdependence between the congestion window (cwnd) size and the transmission RTT of the TCP segment for the TCP version based on a fast retransmit with a fast recovery mechanism has been presented in Figure $3 \mathrm{~b}$. The general idea of a fast retransmit with a fast recovery is not to replace the RTO parameter, but to work in parallel, allowing the sender to retransmit the lost segment even if the timeout has not expired. In a TCP communication, when a segment arrives out of order, the receiver resends the ACK that was last sent, causing the sender to receive duplicate ACKs. This signals to the sender that the segment is either lost or delayed. This causes a reordering of the segments. In the case of segment reordering, it is assumed that only one or two duplicate ACKs will be sent. The case when three ACKs are received by the sender before the RTO has expired indicates that the segment is lost. This triggers a fast retransmit and a fast recovery mechanism (Figure 3b). In the standard implementation of the fast retransmit with a fast recovery phase, the sender, instead of going into the slow-start phase, decreases the ssthresh by one-half of the current cwnd (but by no less than two segments). Additionally, the sender retransmits the missing segment and sets the cwnd to a value equal to ssthresh plus 3 times the segment size [24] (Figure 3b). Each time, the sender receives an additional duplicate ACK after the third cwnd is incremented by MSS. When the ACK that acknowledges the new data arrives, cwnd is set to the new value of ssthresh (which is set at the moment of the initial triggering of the mechanism). It then enters the congestion avoidance phase, as shown in Figure 3b [24].

The aforementioned TCP CC mechanism is the standard implementation of the mechanism. It can vary depending on the CC algorithm used. There are numerous CC algorithms that have been developed and proposed as CC emerged as one of the most studied areas 
of internet research during the last few decades [25]. TCP CC, although not part of the original TCP implementation, has become an essential element of TCP as the entire TCP performance depends on the CC algorithm.

\subsection{TCP CC Algorithms}

CC algorithms are being developed with a focus on optimizing different metrics and making trade-offs between the various metrics in order to accommodate the different working environments and use cases. The main metrics that the Transport Modelling Research Group (TMRG) of the Internet Research Task Force proposed for evaluating CC algorithms were [26]: throughput, delay, packet loss rates, fairness, convergence times and robustness. Depending on the desired working environment, TCP CC algorithms are usually designed to make trade-offs between these metrics. There are three main categories of TCP CC algorithms as shown in Figure 4: (1) loss-based algorithms that use packet loss as an indicator of congestion, (2) delay-based algorithms that predict packet loss based on RTT measurements and (3) hybrid algorithms in which, the loss-based and delay-based methods are combined. Table 1 presents the comparison of the different CC algorithms according to their classification and year of introduction.
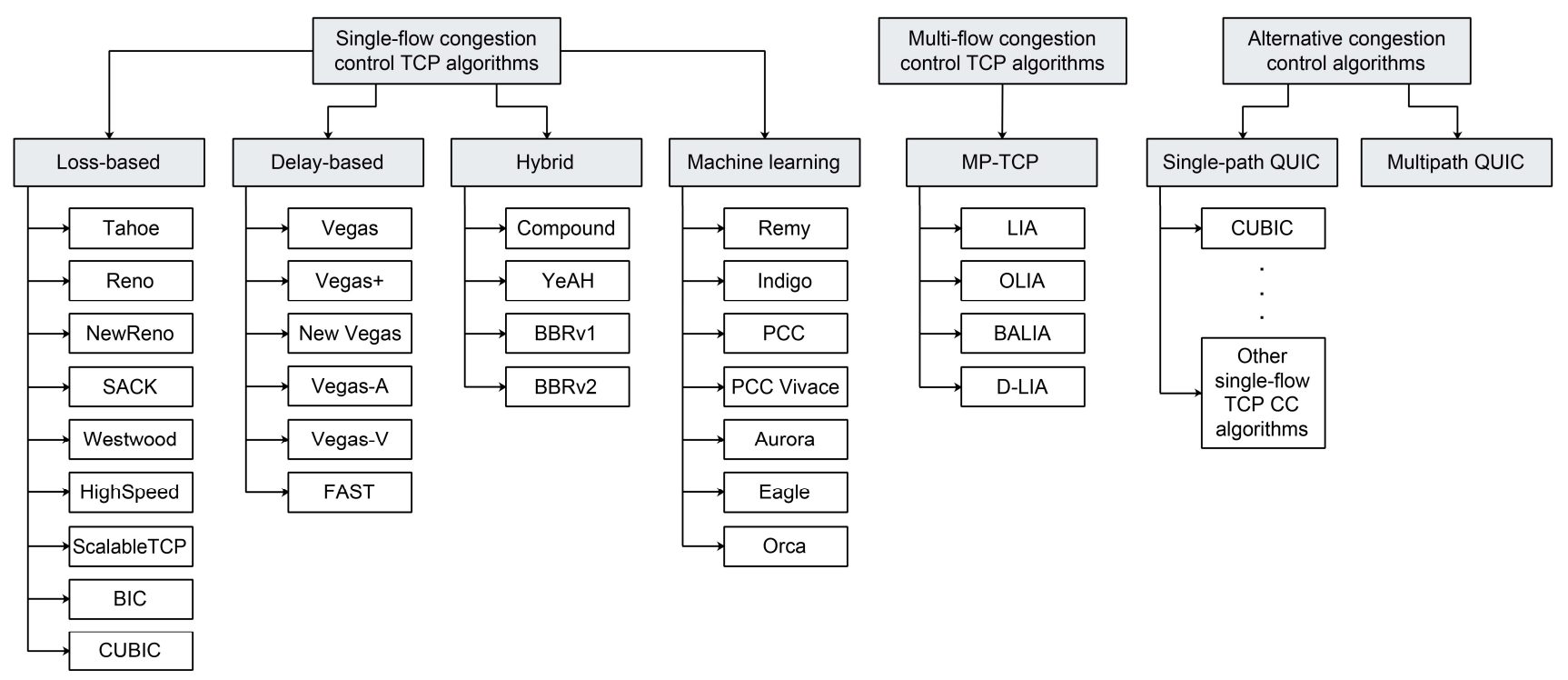

Figure 4. Main categories and types of the TCP CC algorithms.

\subsubsection{Loss-Based TCP CC Algorithms}

A Tahoe TCP was the first CC algorithm that implemented a fast retransmit phase [22], followed by the Reno TCP [27] which included a fast recovery procedure (Table 1). The Tahoe TCP and Reno TCP are loss-based algorithms, and both consider RTO and duplicate ACKs as an indication of segment loss due to network congestion. The main difference between the two CC algorithms is in how they respond after receiving three duplicate ACKs and how they perform the fast retransmitting of the segments. After the fast retransmit phase, the Tahoe TCP switches to the slow start phase, whereas in contrast, the Reno TCP avoids the slow start phase by entering the fast recovery phase. In high-traffic environments where multiple segment losses occur in a single congestion window, the Reno TCP has shown a performance decrease as it can only detect single-segment losses [28]. 
Table 1. Overview of the most relevant single-flow TCP CC algorithms.

\begin{tabular}{|c|c|c|c|}
\hline CC Algorithm & $\begin{array}{l}\text { Introduction } \\
\text { Year }\end{array}$ & Type & Features \\
\hline Tahoe [22] & 1988 & Loss-based & Slow start, congestion avoidance and a fast retransmit mechanism. \\
\hline Reno [27] & 1990 & Loss-based & Introduced fast recovery mechanism. \\
\hline NewReno [29] & 1999 & Loss-based & Fast recovery modification to allow multiple retransmissions. \\
\hline SACK [30] & 1996 & Loss-based & Selective ACK option. \\
\hline Westwood [31] & 2001 & Loss-based & $\begin{array}{l}\text { Introduced a faster recovery mechanism that controls the sending } \\
\text { rate according to the available bandwidth estimation. }\end{array}$ \\
\hline HighSpeed [32] & 2003 & Loss-based & $\begin{array}{l}\text { Introduced a modified TCP response function to allow for a faster } \\
\text { cwnd increase and a faster recovery time in situations with a high } \\
\text { cwnd size. }\end{array}$ \\
\hline Scalable TCP [33] & 2003 & Loss-based & $\begin{array}{l}\text { After each received ACK during the RTT, algorithm increases the } \\
\text { cwnd size in proportion with the defined constants. After the packet } \\
\text { loss decreases cwnd by a smaller factor, the standard CC } \\
\text { is exploited. }\end{array}$ \\
\hline BIC [34] & 2004 & Loss-based & $\begin{array}{l}\text { Uses the binary search increase and additive increase techniques to } \\
\text { determine the cwnd size. }\end{array}$ \\
\hline CUBIC [35] & 2008 & Loss-based & $\begin{array}{l}\text { Uses the cubic function for cwnd control characterised by a steady } \\
\text { state and a maximal probing behaviour. }\end{array}$ \\
\hline Vegas [36] & 1994 & Delay-based & $\begin{array}{c}\text { Modification of TCP Reno that predicts network congestion before } \\
\text { an actual loss of segments occurs. Uses a fine-grained RTT } \\
\text { estimation and has a very efficient segment } \\
\text { retransmission schedule. }\end{array}$ \\
\hline Vegas+ [37] & 2000 & Delay-based & $\begin{array}{l}\text { Modification of TCP Vegas that introduced an aggressive mode to } \\
\text { overcome fairness issues when competing with TCP Reno. }\end{array}$ \\
\hline New Vegas [38] & 2005 & Delay-based & $\begin{array}{l}\text { Implemented three server-side modifications of TCP Vegas to } \\
\text { overcome performance issues in a high latency environment. }\end{array}$ \\
\hline Vegas-A [39] & 2005 & Delay-based & $\begin{array}{l}\text { Implemented modified congestion avoidance mechanism to } \\
\text { address fairness, rerouting and bias against high bandwidth } \\
\text { connections issues of TCP Vegas in wired and satellite networks. }\end{array}$ \\
\hline Vegas-V [40] & 2012 & Delay-based & $\begin{array}{l}\text { Modification of TCP Vegas- } \mathrm{A} \text { that addresses fairness and } \\
\text { aggression issues when competing with TCP Vegas, TCP Vegas-A } \\
\text { and TCP Reno flows. }\end{array}$ \\
\hline FAST [41] & 2004 & Delay-based & $\begin{array}{c}\text { Designed for high-speed long-latency networks. Adjusts the cwnd } \\
\text { according to the feedback information of the average RTT and } \\
\text { average queuing delay. Uses scaling parameters to effectively } \\
\text { utilise the network capacity. }\end{array}$ \\
\hline Compound [42] & 2006 & Hybrid & $\begin{array}{l}\text { Based on the loss-based slow start phase. During the congestion } \\
\text { avoidance phase, it uses a combination of two components, a } \\
\text { standard loss-based and a new scalable delay-based component. }\end{array}$ \\
\hline YeAH [43] & 2007 & Hybrid & $\begin{array}{l}\text { Performs a dynamic exchange between a slow mode during the } \\
\text { congestion avoidance phase and a fast mode during the fast } \\
\text { recovery phase. }\end{array}$ \\
\hline BBRv1 [44] & 2016 & Hybrid & $\begin{array}{l}\text { Builds an explicit model of the network using the estimated RTT } \\
\text { and the estimated available bottleneck bandwidth in order to } \\
\text { prevent congestion. }\end{array}$ \\
\hline BBRv2 [45] & 2018 & Hybrid & $\begin{array}{l}\text { Uses ECN signals, improved fairness with CUBIC and lower packet } \\
\text { loss rates for the optimisation of the TCP CC performance. }\end{array}$ \\
\hline
\end{tabular}


TCP NewReno is an improved version of the TCP Reno algorithm. In order to overcome the performance issues of the TCP Reno, the TCP NewReno introduces a slight modification of the TCP Reno's fast recovery mechanism (Table 1) [29]. After receiving multiple duplicate packets, like its predecessor, the TCP NewReno enters the fast retransmit phase. However, the TCP NewReno does not exit the fast recovery phase until all of the outstanding data (send without received ACK) at the time of entering the fast recovery phase is acknowledged, thus preventing multiple cwnd reductions.

TCP selective acknowledgments (SACKs) have been introduced to overcome the limitations of the algorithms that use cumulative ACKs (Table 1). This approach allows for the retransmission of more than one segment per RTT. In an established TCP connection, the receiver uses the selective ACKs (SACKs) option to inform the sender about all successfully received segments, thus allowing the sender to retransmit only the missing segments in one RTT [30].

TCP Westwood is a CC algorithm that uses a server-side modification of the TCP Reno cwnd control mechanism (Table 1) [31]. TCP Westwood improves the performance of TCP Reno, especially in lossy wireless networks due to its robustness against sporadic wireless network errors. It uses a mechanism called faster recovery where instead of halving cwnd after three duplicate ACKs, the mechanism adjusts the cwnd and ssthresh parameters based on the end-to-end estimation of the available bandwidth. The bandwidth of the connection is estimated through the regular monitoring of the ACKs returning rate. After the congestion event, the size of the cwnd and ssthresh parameters is set according to the bandwidth estimate, thus ensuring a faster recovery as opposed to the TCP Reno response [31].

HighSpeed TCP is a modification of the classical TCP CC mechanism [14] that aims to address the limitations of connections with large congestion windows (Table 1) [32]. In the situation of a small cwnd, the operation of the HighSpeed TCP is the same as in the standard CC. On the other hand, when the current cwnd is greater than the specified parameter, the HighSpeed TCP uses a modified TCP response function that allows for a faster cwnd increase rate and a faster recovery time after packet loss. A variation of the HighSpeed TCP known as the Scalable TCP [33] is optimised for high-speed wide area networks. It is based on a simple modification of the traditional CC mechanism [14]. The main goal of the Scalable TCP algorithm is to update the cwnd in a scalable fashion. This means that the recovery times are proportional only to the RTT of the connection, which makes the algorithm more robust in high BDP networks.

A binary increase congestion control (BIC) TCP algorithm has been developed to address unfairness issues and to provide bandwidth scalability in high-speed networks with large delays (Table 1) [34]. The BIC TCP algorithm uses a binary search increase and additive increase as the two techniques used to determine the cwnd size. Binary search increase is a search technique involving aggressive initial bandwidth probing in a situation where there is a large difference between the current cwnd size and the target cwnd size. The mechanism becomes less aggressive when the current cwnd size gets closer to the target cwnd size. In the case of network congestion, the BIC TCP reduces cwnd from performing multiplicative decreases. After a significant cwnd decrease, the BIC algorithm performs an additive increase scheme by increasing cwnd linearly. The increase then becomes logarithmic [34]. By combining the binary search increase with the additive increase technique, the BIC algorithm ensures RTT fairness and faster convergence times.

The authors of [35] proposed a high-speed TCP variant called CUBIC to overcome the fairness and complexity issues of the previous CC algorithms (such as the TCP BIC). TCP CUBIC has become the dominant CC algorithm on the Internet [46] and it is currently the default algorithm distributed by Linux (Table 1). The performance of the TCP CUBIC algorithm is visualised in Figure 5. To adjust the cwnd size, the TCP CUBIC uses a cubic function that is characterised by the aggressive growth of the window size following the event when a packet loss is detected. When the cwnd size approaches the point of the last congestion event (marked as $W_{\text {max }}$ in Figure 5), it slows its growth to almost zero. After reaching the $W_{\max }$ point, the algorithm slowly probes the network by increasing the cwnd 
size at a slow rate. As it moves away from the $W_{\max }$, it rapidly accelerates its growth rate (Figure 5). Using a cubic function for calculating the cwnd growth rate ensures fairness among the concurrent flows and good performance properties [25].

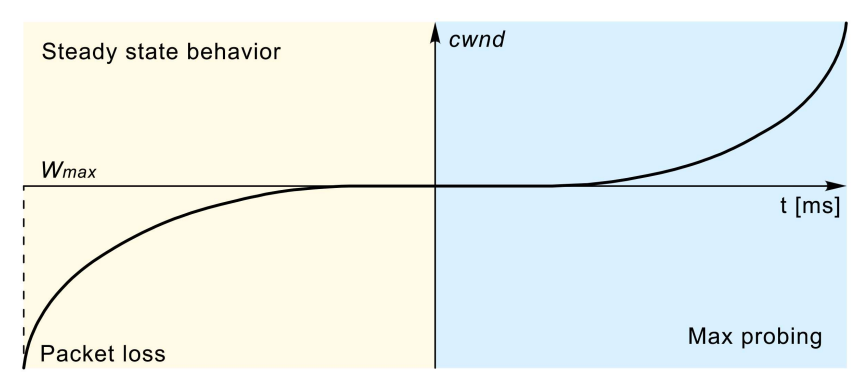

Figure 5. TCP CUBIC window growth function after the packet loss event.

\subsubsection{Delay-Based TCP CC Algorithms}

All aforementioned loss-based CC algorithms use segment loss as a congestion indicator and they are based on utilising a reactive congestion adaptation method. These algorithms are opposed to proactive delay-based CC algorithms (Figure 1) [25]. A typical representative of loss-based CC algorithms is TCP Vegas (Figure 4). This was introduced as a delay-based modification of the TCP Reno algorithm (Table 1). It predicts network congestion before actual segment loss occurs. TCP Vegas uses a fine-grained RTT estimation that increases the accuracy of the computed timeout period, which leads to a very efficient segment retransmission schedule (Table 1) [36]. The TCP Vegas uses a modified retransmission mechanism based on calculating the RTT estimation used for detecting segment loss before waiting for the reception of the three duplicate ACKs (Table 1). This approach increases the algorithm recovery time. It also uses a modified version of the slow start and congestion avoidance phases, which are thoroughly explained in Ref. [36]. The TCP Vegas delay-based approach can improve the overall TCP flow throughput by keeping the sending rate stable. However, due to the slow cwnd growth, this approach can suffer from performance issues in high-speed networks. In networks with concurrent loss-based flows, the TCP Vegas algorithm shows a decrease in performance due to the unfair resource allocation [25].

In the literature, several modifications of the TCP Vegas algorithm have been proposed. They are dedicated to addressing the limitations of bottlenecks during link sharing when many TCP flows are present, and to alleviate the performance issues in the case of links with high latency. A modified version of the TCP Vegas, called the TCP Vegas+ has been introduced to overcome fairness issues between the TCP Reno and the TCP Vegas (Figure 4) [37]. The algorithm introduced an aggressive mode to maintain fair throughput when competing with TCP Reno flows. To address performance issues of TCP Vegas over high latency links, a TCP New Vegas (Figure 4) has also been introduced. Compared with TCP Vegas, TCP New Vegas implements three sender-side modifications of TCP Vegas [38]. Furthermore, another modification of TCP Vegas has been developed, called TCP Vegas-A (Figure 4). The algorithm uses the modified congestion avoidance mechanism of TCP Vegas to address issues such as fairness against TCP New Reno flows, rerouting issues, bias against high bandwidth connections and fairness issues between old and new connections in wired and satellite networks [39]. The authors in Ref. [40] proposed a modified version of TCP Vegas-A, called TCP Vegas-V (Figure 4). This CC algorithm improves performance regarding fairness and aggression features in the network environment containing competing TCP Vegas, TCP Vegas-A and TCP Reno flows.

Another representative of delay-based CC algorithms is the FAST TCP algorithm that is designed for high-speed long-latency networks (Table 1) [41]. Based on the feedback information of the average RTT and average queuing delay, the FAST TCP periodically updates cwnd in order to control the packet transmission. This feedback information is 
provided by a latency estimation of each packet sent. Fairness and the number of buffered packets in the network are both controlled by a scaling parameter. FAST TCP adjusts its cwnd size constantly according to the number of buffered packets. If the number of buffered packets is far from the defined scaling parameter, the FAST TCP algorithm increases or decreases the cwnd size, effectively utilising the network capacity. On the other hand, when the number of buffered packets reaches the target scaling parameter, the FAST TCP algorithm adjusts its cwnd by a small amount, thus ensuring the networks' stability [47].

\subsubsection{Hybrid TCP CC Algorithms}

Hybrid TCP CC algorithms are a combination of loss-based and delay-based algorithms (Figure 4). In a congested network situation or a network with high link utilisation having short bottleneck queues, hybrid algorithms tend to use a delay-based CC approach. On the other hand, in a high-speed network with a low link utilisation, hybrid algorithms tend to use a more aggressive CC approach that is characteristic of loss-based algorithms [20]. Some of the well-known representatives of hybrid CC algorithms are Compound TCP, Yet Another Highspeed (YeAH) TCP, and the TCP Bottleneck Bandwidth and Round-trip Propagation Time (BBR) algorithm (Figure 4).

The Compound TCP algorithm is representative of a hybrid-based CC algorithm developed for high-speed and long-distance networks. It was the default TCP algorithm in Microsoft Windows operating systems (Table 1) [42]. Compound TCP is designed to ensure high network utilisation, RTT and TCP fairness using a combination of loss-based and delay-based approaches. In the slow start phase, Compound TCP has the same aggressive behaviour characteristic as standard loss-based CC algorithms. During the congestion avoidance phase, it uses a combination of a standard loss-based component and a new scalable delay-based component. The delay-based component is based on the TCP Vegas algorithm, and it is controlled by a new state variable called dwnd (delay window). By encompassing the delay-based component, Compound TCP provides faster network utilisation and a reduction of the sending rate according to the bottleneck queue. This ensures better TCP fairness and the reduction of the sending rate in a packet loss event.

YeAH TCP is a hybrid variant of the high-speed TCP CC algorithm which uses two operation modes known as fast and slow modes respectively (Figure 4) [43]. During the fast recovery phase, the fast mode is initiated and the cwnd is incremented aggressively as in the Scalable TCP algorithm (Table 1). In the congestion avoidance phase, the slow mode is triggered by adjusting the cwnd size in the TCP Reno fashion. YeAH TCP uses the number of packets in the bottleneck queue determined through RTT measurements. This packet number is used as a parameter to determine the state of the algorithm. The main benefits of YeAH TCP are its high efficiency in situations where there are small link buffers typical of high BDP networks. An important benefit of the YeAH TCP algorithm compared to loss-based algorithms is its improved fairness.

The TCP BBR algorithm was developed by Google to overcome the problems faced by loss-based CC algorithms such as throughput issues in a small buffer scenario and the bufferbloat problem in a network with large bottleneck buffers (Table 1) [44]. It is a cuttingedge CC algorithm that creates an explicit CC model based on the recent measurements of the RTT and the delivery rate of the network. Using an explicit CC data model, the algorithm adjusts the sending rate accordingly. According to the periodic estimation of the available bandwidth and minimal RTT, the BBR algorithm prevents congestion by ensuring low delay and high throughput operations. The sending rate is determined through the pacing gain process, which is the main mechanism that the BBR uses to control the sending behaviour. This is done by maximising the rate at which the BBR schedules packets. When the bottleneck bandwidth (BtlBw) has been estimated, the BBR deliberately reduces the pacing gain to drain the queues in order to estimate the round trip propagation time (RTprop). Periodical measurements of BDP calculated as the multiplication of the $B t l B w$ and RTprop parameters ensure performance within the Kleinrock optimal operating point [48], where data delivery rate is maximised and delay is minimised [49]. However, 
the initial version of the BBR has drawbacks which are mainly reflected in unfairness when competing with loss-based CC, including a large number of packet losses and increased queuing delays [50]. For that reason, an improved version known as a BBRv2 has been introduced to alleviate these problems [45]. Google has deployed TCP BBR across all of its services, which has resulted in a higher throughput, reduced latency and better overall connection quality. Even though TCP BBR is a relatively new CC algorithm, [46] showed that after TCP CUBIC, it is the second most dominant CC algorithm used on the Internet. It is reasonable to predict that TCP BBR will surpass TCP CUBIC in the near future.

\subsection{TCP Fairness}

In a situation of multiple concurrent TCP flows competing for the same bandwidth on the network link, some TCP CC algorithms may receive more of a bandwidth share than other TCP flows. The challenge of satisfying bandwidth allocation fairness is a serious problem in TCP CC. Hence, one of the main goals that CC algorithms need to accomplish is to ensure fairness among flows that are competing for the same bottleneck bandwidth [51]. Due to the competition among different TCP flows, fair bandwidth allocation among TCP flows can severely degrade the performance of the CC algorithms characterised by a less aggressive approach in the competition for bandwidth.

Loss-based CC algorithms (e.g., TCP Reno) have an aggressive nature as packet loss is the only indicator of congestion. This nature is mainly reflected in the continuous increase of cwnd until packet loss occurs. On the other hand, delay-based algorithms (e.g., TCP Vegas) use RTT estimation to predict network congestion before an actual loss has occurred, reducing the sending rate accordingly. In a network with many competing flows that are loss-based, delay-based flows cannot get their fair throughput share as shown in Ref. [52]. This is because flows with an aggressive loss-based approach will obtain a larger amount of bandwidth than their fair share. Likewise, the fairness issue has been detected between the most dominant CC algorithms on the Internet, and the CUBIC and BBR algorithms are no exception. It has been shown that TCP BBR favours small buffers and gets a significantly higher share when competing with CUBIC. In contrast, when using large buffers, BBR cannot compete with CUBIC because it occupies most of the bottleneck bandwidth as shown in Ref. [50]. When deploying CC algorithms in dynamic environments, such as the mmWave 5G network where a large number of lines of LOS-NLOS transitions are expected, fairness issues should be seriously considered. The NLOS state of the TCP flow causes an increase in RTT, which consequently results in bandwidth unfairness among the flows [53].

\subsection{TCP Optimisation Techniques}

The purpose of TCP CC mechanisms is to address congestion on the network and to fully utilise the network resources. Besides CC, several TCP optimisation techniques have been developed to enhance the operations of CC. To solve the excessive buffering of packets on the network and thus preventing bufferbloat, active queue management (AQM) techniques are proposed as opposed to a basic drop-tail queuing mechanism. AQM is a technique based on proactively discarding packets from the buffer before the queue is full, thus reducing the risk of a queuing delay, preventing the bufferbloat problem and proactively reducing network congestion [54]. AQM schemes are deployed on the network device buffers (e.g., routers, BSs, etc.), rather than as part of a TCP implementation. The benefit of using the AQM is to maintain a small queue size to prevent overflowing the buffers with a large burst of packets. Furthermore, keeping the queue size small reduces the queuing delay and minimises the overall end-to-end delay of the network. Finally, the AQM avoids the impact of global TCP synchronisation and this contributes to the increased throughput and better utilisation of the network [55].

Random early detection (RED) is an example of a basic AQM algorithm that addresses global synchronization problem, minimise network congestion and limits network delay [56]. Due to the configuration difficulties and performance issues regarding bursty traffic of RED, a new technique called controlled delay (CoDel) has been developed. The 
CoDel is an AQM technique [57] that treats differently low delay queues and queues that continuously buffer packets causing the increased delay. CoDel operates by regularly monitoring the minimum queuing delay in specific intervals and by discarding packets when the minimum queue delay is exceeded. By using queue delay instead of buffer queue occupancy as a queue management metric, CoDel improves network utilization, which further contributes to achieving high throughput and better queue management [58].

Another TCP optimisation method that is used in combination with CC algorithms is the explicit congestion notification (ECN) technique. The ECN is a congestion detection technique based on the setting of the codepoint (mark) in the ECN field of the IP packet header [59]. Based on this codepoint mark annotation, the ECN-capable device can signal on the transport layer regarding the incipient congestion before actual congestion occurs. This allows the CC algorithms to adjust their cwnd size accordingly. ECN is generally used in combination with AQM techniques, where ECN marks the IP packet header based on the information obtained from the AQM scheme. Enabling the ECN technique can result in packet loss reduction, queuing delay reduction, and improved throughput in the connection [59]. Instead of dropping packets, an AQM technique may interact with ECN to mark packets and therefore indicate congestion prior to the actual packet loss occurs [60].

Since regular ECN informs the sender only once per RTT about incipient congestion, a more accurate ECN feedback scheme (AccECN) has been proposed. It is based on allowing more than one feedback signal to be transmitted per RTT [61]. Although AccECN was proposed in 2011, it is still an Internet-draft working document expected to be standardised.

\subsection{Multistream TCP Variants and Alternatives}

Although TCP is the dominant transport protocol used today, there are emerging variants and alternatives that can improve overall network performance (Figure 4). The two most relevant protocols are multipath TCP (MP-TCP) and Quick UDP Internet Connection (QUIC). These protocols have been widely used and recently standardized by an Internet Engineering Task Force (IETF). MP-TCP has been introduced as a TCP variant for multipath data communication and QUIC is a UDP-based TCP alternative initially developed for hypertext transfer protocol (HTTP) traffic (Figure 4).

\subsubsection{Multipath TCP}

Standardized by an IETF in 2020, MP-TCP is an extension to the TCP that handles multiple paths simultaneously for a single data stream [62]. Multipath connection is envisioned to utilize the presence of multiple network interface cards (NICs) commonly found in today's devices (e.g., smartphones). Hence, MP-TCP can use multiple network interfaces (e.g., 4G, 5G, Wi-Fi, Ethernet, etc.) to create multiple subflows that use multiple paths for a single connection. Using a multipath approach for end-to-end communication can improve utilization of network resources, enhance throughput, and ensure more robust and resilient communication [62].

In Figure 6, a comparison between the TCP protocol stack and the MP-TCP protocol stack has been presented. It can be seen that MP-TCP divides a single TCP connection into multiple different TCP flows. This dividing requires a CC algorithm that can control the transmission rate for each subflow. To control the data transmission rate for each subflow, MP-TCP CC must satisfy three design goals related to ensuring fair bottleneck bandwidth usage and robustness of the connection [63]. First, multiple subflows of a single multipath connection should perform at least as well as a single path connection would on the best path available. Second, multipath connection should not use more capacity than a single path TCP connection. Finally, an MP-TCP should balance congestion in such a way as to move data towards paths that are less congested. 

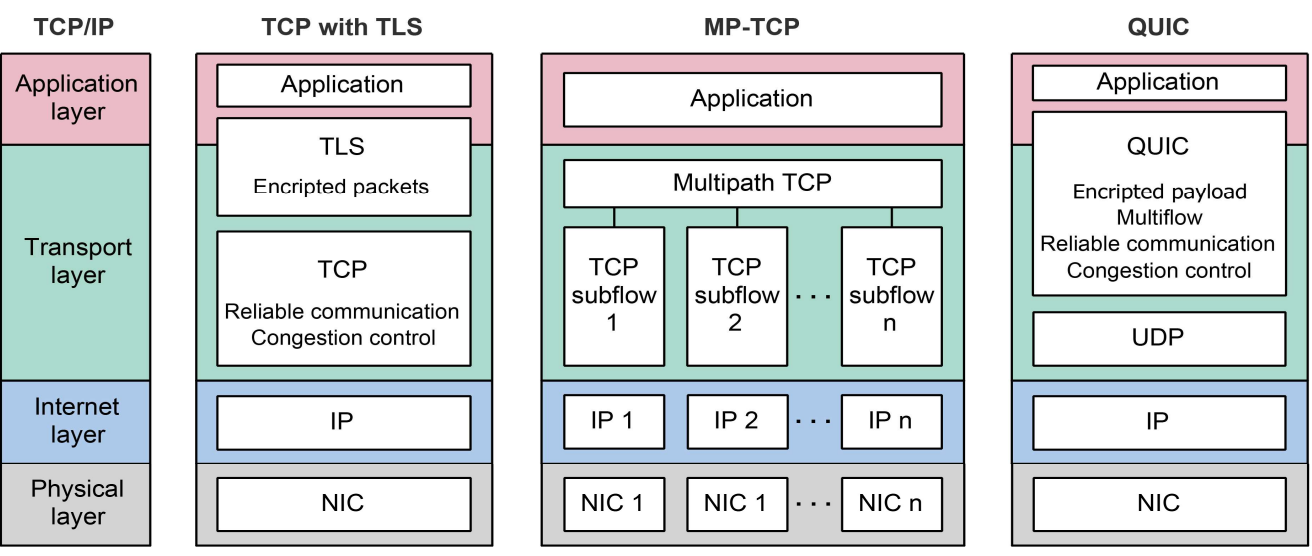

Figure 6. Comparison between TCP with TLS, QUIC and MP-TCP protocol stacks.

Depending on the cwnd control method for each subflow, CC mechanisms used with MP-TCP are characterized as uncoupled or coupled. The uncoupled CC mechanism handles each subflow independently allowing each subflow to have its own instance of TCP $\mathrm{CC}$ algorithm. On the other hand, the coupled CC mechanism manages cwnd in a coupled manner, by considering the characteristics of all other subflows. The initial coupled CC algorithm (Figure 4), called the linked increases algorithm (LIA) defined in Ref. [63], suffers from performance issues. As demonstrated in Ref. [64], LIA transmits a large amount of data over paths that are congested and can have aggressive behavior with respect to a legacy single-path TCP. To address these issues (Figure 4), the authors in Ref. [64] have proposed the opportunistic linked increases algorithm (OLIA). Additionally, authors in Ref. [65] (Figure 4), proposed a balanced linked adaptation algorithm (BALIA). The performance of BALIA is based on balancing responsiveness, TCP friendliness and cwnd oscillations. However, these algorithms are based on the legacy TCP Reno CC algorithm and follow the AIMD scheme, and as such, they cannot satisfy previously presented three design goals for operation in a 5G mmWave environment [66]. Since the aforementioned coupled CC algorithms are primarily focused on the increase of the cwnd, while neglecting the cwnd decreasing mechanism [67] (Figure 4), authors in Ref. [68] proposed a loss-based MP-TCP CC algorithm called Dynamic-LIA (D-LIA). Instead of halving the cwnd after packet lost occurrence, the D-LIA decreases the cwnd by a dynamically determined factor that depends on the interval between each packet loss [68]. Using this approach, cwnd can reach its optimal size much faster than the regular the TCP AIMD mechanism and thus reduce overall network latency and better utilize network resources. Although the D-LIA achieves better overall performance in terms of throughput and fairness, the authors have detected a downside related to increased packet retransmissions [68].

\subsubsection{QUIC Protocol}

The quick UDP Internet connections (QUIC) protocol is initially developed by Google as an alternative to TCP (Figure 4). It uses user datagram protocol (UDP) at the transport layer (Figure 6). Although standardized in May 2021 by IETF, initial implementation and deployment started in 2012 and QUIC today represents the authenticated and encrypted by default Internet transport protocol with the tendency of eventually replacing TCP and Transport Layer Security (TLS) protocols on the web [69] (Figure 6). QUIC is designed to overcome CC issues of transport and application layer for web-based applications. An upcoming third version of the hypertext transfer protocol (HTTP/3) is designed with QUIC as a built-in transport layer protocol [70] and all major web browsers are starting to support it.

According to Figure 6, QUIC includes the TLS layer with its own framing. This ensures permanent authentication and encryption of the connection and makes the initial connection establishment faster. The handshake messages exchange for TCP with TLS 
and QUIC have been presented in Figure 7. It can be seen that the QUIC handshake only requires one round-trip between client and server to complete, while joint TCP with TLS handshakes requires two round-trips.

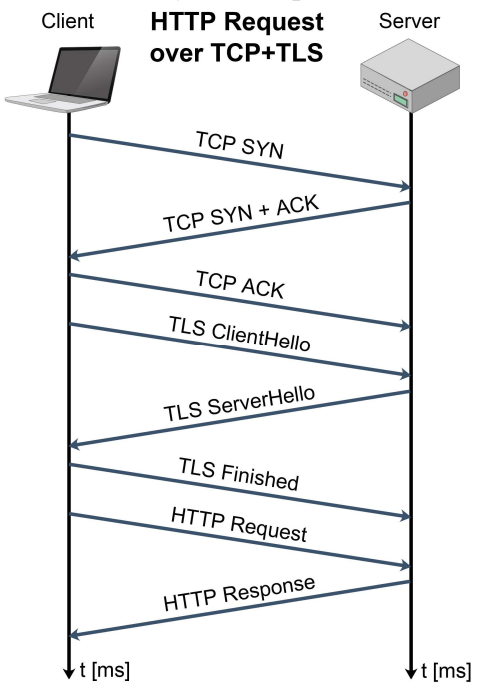

(a)

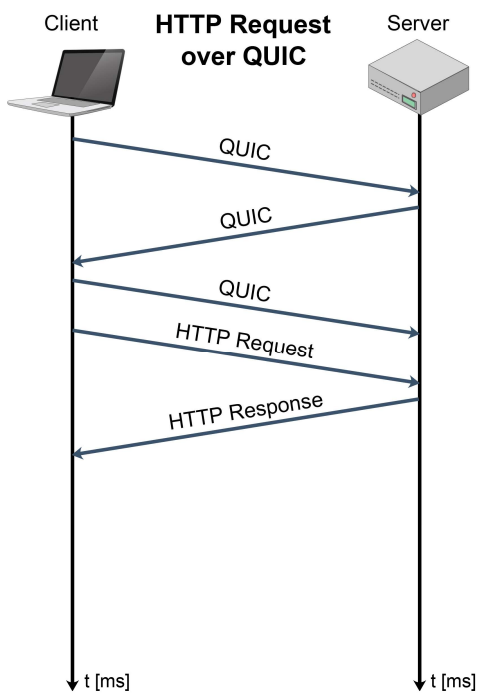

(b)

Figure 7. Comparison of connection establishment and message exchange for: (a) TCP with TLS, (b) QUIC protocol.

The main advantages of QUIC over TCP include: 0-RTT connection establishment which significantly reduces latency, improves $\mathrm{CC}$ with loss detection and minimize headof-line-blocking delay by supporting the multiplexed operation. Furthermore, QUIC has introduced a pluggable CC interface that provides more flexibility over TCP. Namely, QUIC uses generic CC signaling that can support different CC algorithms, thus allowing flexible algorithm selection [71]. Additionally, QUIC uses monotonically increasing packet numbers to ensure proper packet order. This ensures avoiding retransmission ambiguities and simplified loss detection. It also includes information about the delay between the receipt of a packet and the acknowledgment (ACK) sent for that packet, thus allowing for a more precise RTT estimation. To reduce the packet losses caused by packet bursts, QUIC uses a packet pacing mechanism that evenly spaces packet transmissions over time. It has been shown in Refs. [72,73] that a packet pacing mechanism minimizes the probability of packet losses and supports data stream multiplexing. This eliminates head-of-lineblocking problems what can be especially beneficial over lossy wireless environments (e.g., 4G, 5G networks).

Motivated by the development of the MP-TCP protocol, authors in Ref. [74] have proposed a multipath QUIC (MP-QUIC) protocol (Figure 4). The main capability of MPQUIC is the possibility to pool resources for a connection that uses multiple paths and to improve resistance to connection failures. This is especially important for today's multihomed devices (e.g., smartphones) that need to be able to make an uninterrupted switch between different network interfaces. Multipath extension for QUIC (MP-QUIC) was introduced in 2017 and is described in detail in the IETF Internet draft document, which is currently in the process of standardization [75].

\section{Related Work on TCP CC Algorithms in 5G mmWave Networks}

The performance of the $5 \mathrm{G}$ network largely depends on the transport protocol that provides reliable end-to-end communication. As TCP is the most commonly used transport protocol, it has become a necessity for TCP to adapt to new use cases and the requirements of $5 \mathrm{G}$ cellular networks. In this section, we have investigated the algorithms used in the 
most relevant simulation studies for TCP CC in the 5G networks. A comparison of the analysed approaches is presented in Table 2.

In Ref. [76], the authors analysed the TCP CC performance in an end-to-end simulation within different scenarios using the mmWave ns-3 module [77]. In one of the analysed scenarios, the authors simulated the performance of the TCP NewReno algorithm for a mobile user download in a blockage event situation caused by buildings of different sizes.

Table 2. Review of the research articles on CC algorithms in 5G networks.

\begin{tabular}{|c|c|c|c|}
\hline Simulation Scenarios & $\begin{array}{l}\text { Evaluated TCP CC } \\
\text { Algorithms }\end{array}$ & Main Algorithm Drawbacks & Summary \\
\hline \multirow{2}{*}{$\begin{array}{l}\text { UE experiencing LOS to } \\
\text { NLOS transitions and outage } \\
\text { events between mmWave BS } \\
\text { in small buildings and large } \\
\text { building scenarios [76]. }\end{array}$} & NewReno & $\begin{array}{l}\text { Large buffer: bufferbloat. } \\
\text { Small buffer: early } \\
\text { buffer overflow. } \\
\text { Long outage: throughput } \\
\text { degradation and slow } \\
\text { throughput recovery. }\end{array}$ & \multirow{2}{*}{$\begin{array}{l}\text { Evaluated loss-based CC } \\
\text { algorithms showed slow } \\
\text { reaching of full throughput, } \\
\text { large data rate drops, } \\
\text { increased latency and slow } \\
\text { throughput recovery. }\end{array}$} \\
\hline & CUBIC & $\begin{array}{l}\text { Long outage: throughput } \\
\text { degradation and slow } \\
\text { throughput recovery. }\end{array}$ & \\
\hline \multirow[t]{2}{*}{$\begin{array}{l}\text { UE experiencing blockages } \\
\text { from other humans and from } \\
\text { buildings [78]. }\end{array}$} & Cubic with AQM CoDel & $\begin{array}{l}\text { Human blockage: packet drops, } \\
\text { slow throughput recovery. } \\
\text { Building blockage: multiple } \\
\text { packet drops resulting in } \\
\text { near-zero throughput. }\end{array}$ & \multirow{2}{*}{$\begin{array}{l}\text { AQM CoDel does not mitigate } \\
\text { the bufferbloat problem and } \\
\text { DRW showed a much higher } \\
\text { throughput and negligible } \\
\text { oscillation in the delays. }\end{array}$} \\
\hline & CUBIC with DRW & $\begin{array}{l}\text { Low delay and much higher } \\
\text { throughput in both scenarios. }\end{array}$ & \\
\hline \multirow{4}{*}{$\begin{array}{l}\text { High-speed train scenario } \\
\text { with different buffer sizes and } \\
\text { a dense urban scenario, using } \\
\text { remote server and edge server } \\
\text { deployment [13]. }\end{array}$} & NewReno & Remote server: lowest goodput. & \multirow{4}{*}{$\begin{array}{l}\text { Latency is greatly reduced for } \\
\text { all observed CC algorithms } \\
\text { using edge server deployment. } \\
\text { Applying the AQM scheme } \\
\text { with loss-based CC algorithms } \\
\text { can reduce the latency in large } \\
\text { buffer deployments. }\end{array}$} \\
\hline & CUBIC & Edge server: lowest goodput. & \\
\hline & HighSpeed & $\begin{array}{l}\text { Big buffer: high latency } \\
\text { and goodput. }\end{array}$ & \\
\hline & BBR & $\begin{array}{l}\text { Big buffer: high latency } \\
\text { and goodput. } \\
\text { Small buffer: low latency with } \\
\text { weak goodput reduction. }\end{array}$ & \\
\hline
\end{tabular}


Table 2. Cont.

\begin{tabular}{|c|c|c|c|}
\hline Simulation Scenarios & $\begin{array}{l}\text { Evaluated TCP CC } \\
\text { Algorithms }\end{array}$ & Main Algorithm Drawbacks & Summary \\
\hline \multirow{7}{*}{$\begin{array}{l}\text { UE experiencing blockages } \\
\text { between mmWave BS in } \\
\text { extensive blockages, medium } \\
\text { blockages and multiple short } \\
\text { blockages scenarios. } \\
\text { Handover scenario between } \\
\text { three BSs and a mobile user } \\
\text { experiencing multiple short to } \\
\text { extensive blockages. Dense } \\
\text { small cell deployment with } \\
\text { various obstacles in a situation } \\
\text { of multiple BSs serving } \\
\text { multiple UEs when short } \\
\text { flows and background traffic } \\
\text { coexist [12]. }\end{array}$} & NewReno & $\begin{array}{l}\text { Blockage events: Slow full } \\
\text { throughput reach after multiple } \\
\text { losses and slow network probing } \\
\text { in the congestion } \\
\text { avoidance phase. }\end{array}$ & \multirow{7}{*}{$\begin{array}{c}\text { Blockage events greatly } \\
\text { impact latency for loss-based } \\
\text { CUBIC and Scalable TCP. } \\
\text { Delay-based Vegas showed } \\
\text { the lowest throughput with } \\
\text { minimal latency variability. } \\
\text { Hybrid CC algorithms } \\
\text { showed minimal performance } \\
\text { variations. } \\
\text { Loss-based CUBIC showed } \\
\text { high-performance variations } \\
\text { in longer NLOS periods as } \\
\text { opposed to hybrid YeAH } \\
\text { which showed minimal } \\
\text { throughput variations and } \\
\text { required fewer transmissions, } \\
\text { but achieved less throughput } \\
\text { compared to CUBIC. }\end{array}$} \\
\hline & CUBIC & $\begin{array}{l}\text { Blockage events: High RTT } \\
\text { variability in } \\
\text { LOS-NLOS transitions. } \\
\text { Handover: fast throughput } \\
\text { recovery from the slow start. } \\
\text { Multiple flows: high number of } \\
\text { retransmissions and high buffer } \\
\text { occupancy, high throughput. }\end{array}$ & \\
\hline & Scalable TCP & $\begin{array}{l}\text { Blockage events: High RTT } \\
\text { variability in } \\
\text { LOS-NLOS transitions. }\end{array}$ & \\
\hline & Vegas & $\begin{array}{l}\text { Blockage events: Low throughput } \\
\text { with minimal RTT variability. }\end{array}$ & \\
\hline & Westwood & $\begin{array}{l}\text { Blockage events: Slow network } \\
\text { probing in congestion } \\
\text { avoidance phase. }\end{array}$ & \\
\hline & YeAH & $\begin{array}{l}\text { Blockage events: Low RTT and } \\
\text { minimal performance variability. } \\
\text { Handover: slow throughput } \\
\text { recovery from slow mode. } \\
\text { Multiple flows: low number of } \\
\text { retransmissions and } \\
\text { high robustness. }\end{array}$ & \\
\hline & BBR & $\begin{array}{l}\text { Blockage events: Low RTT in all } \\
\text { scenarios and minimal } \\
\text { performance variability. }\end{array}$ & \\
\hline \multirow{4}{*}{$\begin{array}{l}\text { Multiple BSs serving multiple } \\
\text { vehicles moving at random } \\
\text { speed in the mmWave CVNs } \\
\text { environment using two } \\
\text { different mobility models in } \\
\text { rural and urban areas [79]. }\end{array}$} & CUBIC & High cwnd size variability. & \multirow{4}{*}{$\begin{array}{l}\text { Due to the high channel } \\
\text { fluctuations caused by } \\
\text { mobility in CVNs, the } \\
\text { RTW-TCP outperformed the } \\
\text { existing CC algorithms as they } \\
\text { cannot distinguish between } \\
\text { congestion and link failures. }\end{array}$} \\
\hline & Compound & $\begin{array}{l}\text { High average RTT, lowest } \\
\text { aggregate throughput and high } \\
\text { cwnd size variability. }\end{array}$ & \\
\hline & X-TCP & $\begin{array}{l}\text { Low average RTT and high cwnd } \\
\text { size variability. }\end{array}$ & \\
\hline & RTW-TCP & $\begin{array}{l}\text { Low throughput reduction due to } \\
\text { mobility, low RTT and cwnd } \\
\text { continued to increase } \\
\text { despite blockages. }\end{array}$ & \\
\hline \multirow{3}{*}{$\begin{array}{l}\text { Multiple UEs communicating } \\
\text { with single mmWave access } \\
\text { point under static link, short } \\
\text { blockages, long blockages, } \\
\text { and mobility and blockages } \\
\text { scenarios [80]. }\end{array}$} & CUBIC & $\begin{array}{l}\text { Long queuing delay and good } \\
\text { fairness. }\end{array}$ & \multirow{3}{*}{$\begin{array}{l}\text { CUBIC showed a dramatic } \\
\text { increase in the delays in } \\
\text { NLOS conditions. BBR is not } \\
\text { suitable for uninterrupted } \\
\text { high-speed applications and } \\
\text { Prague has fairness issues. }\end{array}$} \\
\hline & BBR & $\begin{array}{l}\text { Low queuing delays and } \\
\text { good fairness. } \\
\text { Periodically reducing } \\
\text { sending rate. }\end{array}$ & \\
\hline & $\begin{array}{c}\text { Prague with DualQ, AQM } \\
\text { and AccECN }\end{array}$ & $\begin{array}{l}\text { Lowest queuing delay and } \\
\text { poor fairness. }\end{array}$ & \\
\hline
\end{tabular}


Table 2. Cont.

\begin{tabular}{|c|c|c|c|}
\hline Simulation Scenarios & $\begin{array}{l}\text { Evaluated TCP CC } \\
\text { Algorithms }\end{array}$ & Main Algorithm Drawbacks & Summary \\
\hline \multirow{5}{*}{$\begin{array}{l}\text { Single gNB serving mobile } \\
\text { users in a small building and } \\
\text { large buildings scenario [81]. }\end{array}$} & NewReno & Lowest performance. & \multirow{5}{*}{$\begin{array}{l}\text { D-TCP using cross-layer } \\
\text { implementation to obtain } \\
\text { SINR information showed the } \\
\text { best performance among the } \\
\text { evaluated CC algorithms. }\end{array}$} \\
\hline & $\mathrm{BIC}$ & $\begin{array}{l}\text { Relatively fast achieves } \\
\text { full throughput. }\end{array}$ & \\
\hline & CUBIC & Long network probing. & \\
\hline & BBR & $\begin{array}{l}\text { Relatively fast achieves } \\
\text { full throughput. }\end{array}$ & \\
\hline & D-TCP & $\begin{array}{l}\text { The best performance and almost } \\
\text { instantly achieves } \\
\text { full throughput. }\end{array}$ & \\
\hline
\end{tabular}

The authors evaluated the different situations in this scenario by adjusting the data rates and radio link control (RLC) buffer sizes. The results show that a large buffer induces a severe latency increase due to the bufferbloat problem, especially in a situation where there is a high data rate. In the case of a reduced RLC buffer size, the results have shown that the buffer is too small to accept arriving packets, causing a large number of packet drops. Furthermore, the triggered fast retransmit phase required a very long time period to retransmit the dropped packets, resulting in very poor performance of the TCP NewReno algorithm when a small buffer size was used. The authors conducted another experiment where they observed how the TCP NewReno and CUBIC algorithms reacted to outage events caused by a large building. In a short outage event, both NewReno and CUBIC recovered capacity almost instantaneously due to the lower layer retransmissions. In the long outage event causing RTO expiration, both NewReno and CUBIC showed a slow throughput recovery due to the small cwnd size in the slow start phase.

The authors in Ref. [78] studied further issues related to the bufferbloat problem (Table 2). Their analyses are based on simulations using the AQM and proposed dynamic receive window (DRW) mechanisms as potential solutions to the bufferbloat problem (Table 2). The research study compared the performance of the CoDel AQM technique and the cross-layer dynamic receive window adaptation technique over mmWave links. The proposed dynamic receive window mechanism uses a cross-layer design to better estimate the receiver window value, which is dynamically updated based on the optimal BDP [78]. In this simulation study, the authors showed that bufferbloat poses a real problem in the mmWave environment that conventional AQM schemes are unable to address. CoDel $A Q M$ in the mmWave environment can reduce latency but it cannot achieve the desired throughput. On the other hand, the authors showed that the proposed cross-layer dynamic receive window scheme can successfully reduce the delay. The delay can be reduced by providing higher channel utilisation without throughput losses [78].

In Ref. [13] the authors conducted a simulation study of TCP in mmWave 5G networks using the four most common CC algorithms: TCP NewReno, HighSpeed TCP, TCP CUBIC and TCP BBR (Table 2). The simulation was performed using the ns-3 mmWave module. For the purpose of the simulation, two challenging scenarios were considered, specifically a high-speed train and a dense urban scenario. The simulations also took into account the edge server and remote server deployment focusing on goodput (application-level throughput) and latency. In the high-speed train scenario, the authors analysed TCP CC's performance using different RLC buffer sizes and MSSs for the fast-moving UEs served by multiple mmWave NR gNodeB (gNB) base stations. In the dense urban scenario, the authors evaluated TCP CC's performance where different static UEs are served by a single mmWave gNB. The simulations were performed for the LOS and NLOS conditions using standard MSS and RLC buffer sizes. The authors concluded that moving the server closer to the network's edge can dramatically improve the latency for all observed scenarios. It was shown that using large buffers can have a positive impact on goodput, but that it 
can also introduce the bufferbloat problem, causing a latency increase. Conversely, using small buffers provides a lower latency at the expense of a lower goodput induced by the buffer overflow. A trade-off between bandwidth and latency needs to be considered to find the optimal solution. Furthermore, it is shown that the use of a larger MSS size for loss-based CC algorithms can have a remarkable impact on goodput due to the reduced time to reach the full link rate and the rapid recovery after a congestion event. Finally, due to the same buffer size existing for the different channel conditions and network latencies in the dense urban simulation scenario, the authors observed high RTT variability among the CC algorithms.

The authors in Ref. [12] analysed the performance of the TCP CC algorithms in the mmWave network through extensive simulations using the ns- 3 mmWave simulation module. Seven different CC algorithms were analysed in a single flow scenario involving different blockage events (Table 2). The authors adjusted the RLC buffer to $7 \mathrm{MB}$ and RTO to $200 \mathrm{~ms}$ for the purpose of the simulation. It has been shown that adjusting the RLC and RTO values can have a great impact on all CC algorithms, thus achieving maximum throughput and faster recovery times. The analysis has shown that the Scalable TCP and the CUBIC CC algorithms achieve fast recovery times for short NLOS periods, as opposed to the situation of LOS to extensive NLOS transitions where their performance considerably degrades. On the other hand, the Westwood and the NewReno CC algorithms are shown to be less susceptible to the LOS-NLOS transitions, exhibiting fewer fluctuations in the RTT metrics. The YeAH and BBR algorithms performed similarly, showing robustness in the LOS-NLOS transitions, whereas the BBR exhibited the lowest RTT values among all scenarios (Table 2). The Vegas algorithm has shown a good level of performance regarding RTT at the price of the lowest throughput between the observed CC algorithms. In the second scenario, the authors simulated a single flow with a handover scenario where the mobile user is experiencing short to extensive blockages using the CUBIC and YeAH CC algorithms (Table 2). The simulation showed that, in the case of rapid handover events, the loss-based CUBIC algorithm achieved link capacity quickly whereas the hybrid YeAH performed significantly slower. In the third scenario, the authors analysed the performance of the CUBIC and YeAH algorithms, simulating a scenario of multiple concurrent flows where 10 users were served by 4 BSs (Table 2). Specifically, the short data flows with a background traffic coexistence were simulated with respect of the number of retransmissions and the RLC buffer occupancy [12]. It was shown that the YeAH algorithm outperformed the CUBIC in a number of retransmissions for every usage scenario except one. In the situation where the transmitted data is relatively small, all analysed CC algorithms performed similarly, achieving significantly lower data rates. Regarding the RLC buffer occupancy, in the absence of background transactions with long flows, both CUBIC and YeAH performed similarly. This is as opposed to the situation involving the existence of long flow transactions in the background where CUBIC achieved higher data rates and a higher buffer occupancy.

New 5G technology supporting mmWave communications will enable a vast number of applications in various industries. The idea of connected vehicles has become more feasible today as new mobile technology emerges. In Ref. [79], the authors compared the performance of conventional TCP CC algorithms in mmWave connected vehicular networks (CVNs) with the proposed real-time wireless TCP (RTW-TCP) CC algorithm (Table 2). It was shown that the high data rates required by CVNs, along with reliable end-to-end communication, represent critical requirements that need to be addressed appropriately. Since the TCP cannot distinguish between channel issues and network congestion, the authors in Ref. [79] detected blockages and beam misalignment as potential issues in mmWave communications in CVNs. The existing CC algorithms, such as Compound TCP, TCP CUBIC, the cross-layer approach to TCP uplink flows (X-TCP) and the proposed RTW-TCP were analysed in various simulation scenarios (Table 2). The proposed TCP mechanism is based on TCP CUBIC with a difference in situation when packet loss occurs. RTW-TCP adjusts its cwnd size according to the vehicle mobility and channel 
quality information (CQI). It reduces cwnd size in long blockage situations and maintains cwnd size in short blockage situations. The results revealed that RTW-TCP outperforms the other CC algorithms in every simulation scenario in terms of the higher achieved throughput and shorter RTT. The authors concluded that the successful implementation of TCP in mmWave CVNs mandates distinguishing between network congestion and link failures. To achieve the required performance, the algorithm requires adjusting the cwnd size accordingly. Finally, the authors noted that the channel characteristics of the mmWave band present a much bigger issue in future CVNs than network congestion. This is the consequence of the fact that congestion will be significantly reduced due to the improved hardware and wider channel bandwidth.

Low latency applications are one of the three main 5G usage scenarios that are expected to be implemented in the mmWave frequency bands. However, as mmWave frequencies are highly susceptible to blockages caused by various objects (e.g., buildings, vehicles, trees) and even the human body, which significantly increases the network delay causing bufferbloat, the use of these frequencies poses a great challenge for low-latency applications [80]. As traditional loss-based CC algorithms are unable to overcome buffer problems, the authors in Ref. [80] conducted an experimental evaluation to examine the behaviour of low-delay CC algorithms in highly variable environments (Table 2). For the purpose of the evaluation purpose, they conducted a CloudLab [82] testbed experiment to explore the behaviour of two low-latency CC algorithms. The TCP BBR and TCP Prague algorithms were compared to the TCP CUBIC algorithm (Table 2). The TCP Prague CC algorithm is part of the low latency, low loss, scalable throughput architecture and it is not a standalone CC algorithm [83]. Four different mmWave link conditions were evaluated in this experiment, including static link, short blockages, long blockages, and mobility with a blockage condition. The results in Ref. [80] confirm that TCP CUBIC is unable to overcome the delay problem in the mmWave environment. Furthermore, in the case of blockages, the ECN-based TCP Prague algorithm shows an increase in delay while still maintaining a lower delay compared to the CUBIC algorithm. It therefore provides good queuing delay management. On the other hand, despite the lack of ECN support, the TCP BBR algorithm maintains low latencies regardless of the link conditions. Moreover, due to the periodical decrease of the cwnd size for the purpose of the minimum RTT path estimation, the TCP BBR algorithm may not perform well in applications that require an uninterrupted high-speed service. Regarding the throughput fairness of competing flows, the authors have shown that the TCP Prague algorithm has fairness issues, whereas TCP BBR shows that there is a fair throughput share between the concurrent flows. Finally, the authors noticed that TCP Prague's deployment, due to the AccECN scheme on both sides of the communication edges, imposes demanding implementation at a large-scale on the public Internet.

The highly variable channel conditions in the mmWave band and the lack of the TCP CC algorithm that can completely alleviate the impact of channel fluctuations on CC performance have led the authors in Ref. [81] to introduce a new Dynamic TCP (D-TCP) CC algorithm (Table 2). D-TCP is an enhanced TCP CC algorithm specifically designed for mmWave $5 \mathrm{G}$ networks. D-TCP estimates the available bandwidth to control the cwnd considering both the traffic intensity and the varying signal to interference and noise ratio (SINR) fluctuations of the channel. To obtain the SINR information at the transport layer, cross-layer implementation was performed. The authors used an adaptive increase/adaptive decrease (AIAD) paradigm based on the calculated CC factor for adjusting the cwnd. In the situation of packet losses, the D-TCP algorithm restored cwnd to the previous level with the help of the information related to the SINR variations. Overall, this innovative approach to bandwidth estimation resulted in better network utilisation.

The authors in Ref. [81] have analysed and compared the performance of the D-TCP with the NewReno, BIC, CUBIC and BBR algorithms using the mmWave ns-3 module (Table 2). The simulations were performed for two different scenarios. They were based on a mobile user served by a single gNB experiencing blockages in small and large building 
scenarios. In the small building scenario, during the LOS visibility conditions, all CC algorithms performed the same. However, in the situation where the UE experienced NLOSLOS transitions, there were significant performance differences between the observed CC algorithms. The D-TCP algorithm instantly restored the full bandwidth while TCP BBR and TCP BIC achieved the maximum bandwidth relatively fast. However, TCP CUBIC conducted longer network probing to reach the maximum throughput and TCP NewReno performed the worst regarding the recovery time and achieved throughput. In the large buildings scenario, where the UE is experiencing longer NLOS transmissions, the results showed that the best performance was by D-TCP, which almost instantly reached maximum throughput. This is a consequence of the D-TCP property of adapting to the varying SINR fluctuations in the channel. TCP BBR and TCP BIC performed relatively fast, whereas TCP CUBIC demonstrated a worse result compared to the previous simulation. Finally, TCP NewReno has been shown to have a very poor level of performance.

The presented reach in the related work shows that there is no optimal TCP CC algorithm that can be universally used in $5 \mathrm{G}$ mmWave networks. However, different technologies envisioned to be implemented in 5G mmWave networks will impose additional challenges in relation to the realisation of TCP CC. These challenges are discussed in the next section.

\section{Future Challenges in the Realisation of TCP CC for 5G mmWave Networks}

To accommodate the high $5 \mathrm{G}$ requirements and complex novel use cases (Figure 1), various new or adapted features and functionalities have been proposed for the practical implementation of $5 \mathrm{G}$ networks. These features and functionalities can affect the TCP performance and therefore the user experience. In this section, a brief presentation of the most relevant $5 \mathrm{G}$ network features and functionalities is given with a description of their impact on the end-to-end TCP performance (Table 3).

Table 3. Challenges and possible solutions to the realisation of TCP CC with respect to 5G main mmWave network functions.

\begin{tabular}{|c|c|c|c|c|}
\hline $\begin{array}{c}\text { mmWave 5G } \\
\text { Network Function }\end{array}$ & $\begin{array}{c}\text { Reasons for } \\
\text { Implementation in } \\
\text { 5G Networks }\end{array}$ & $\begin{array}{c}\text { Realisation } \\
\text { Challenges in the } \\
\text { 5G Network }\end{array}$ & $\begin{array}{c}\text { Realisation Challenge } \\
\text { Concerning TCP } \\
\text { Performance }\end{array}$ & $\begin{array}{l}\text { A Possible Solution to the } \\
\text { Challenge }\end{array}$ \\
\hline $\begin{array}{l}\text { Frequent horizontal } \\
\text { and vertical } \\
\text { handovers }\end{array}$ & $\begin{array}{l}\text { Reduces outage } \\
\text { occurrence and } \\
\text { blockage occurrence. } \\
\text { Improves network } \\
\text { energy efficiency, UE } \\
\text { signal strength or } \\
\text { BS capacity. }\end{array}$ & $\begin{array}{l}\text { A lot of temporary } \\
\text { disconnections and } \\
\text { connections may } \\
\text { happen in } \\
\text { the network. }\end{array}$ & $\begin{array}{l}\text { Frequent handovers } \\
\text { can confuse the TCP } \\
\text { when scaling its } \\
\text { congestion window } \\
\text { size. This reduces the } \\
\text { capability of TCP to } \\
\text { ensure low } \\
\text { packet drops. }\end{array}$ & $\begin{array}{l}\text { Ensuring appropriate } \\
\text { throughput levels and } \\
\text { TCP CC through the } \\
\text { development of optimal } \\
\text { handover algorithms. } \\
\text { - Implementation of } \\
\text { devices with multi } \\
\text { NICs and CC with } \\
\text { multipath TCP } \\
\text { protocols. }\end{array}$ \\
\hline $\begin{array}{l}\text { Usage of high } \\
\text { frequencies in the } \\
\text { mmWave spectrum }\end{array}$ & $\begin{array}{l}\text { Transmission at } \\
\text { higher frequencies } \\
\text { ensures higher } \\
\text { throughputs. }\end{array}$ & $\begin{array}{l}\text { A blockage occurs } \\
\text { since high } \\
\text { frequencies (in } \\
\text { mmWave spectrum) } \\
\text { cannot pass } \\
\text { through obstacles. }\end{array}$ & $\begin{array}{l}\text { Blockages can cause the } \\
\text { frequent triggering of } \\
\text { TCP RTOs, longer RTTs } \\
\text { and increase the } \\
\text { probability of packet } \\
\text { losses. When compared } \\
\text { with moving UEs, these } \\
\text { negative effects can be } \\
\text { more evident for static } \\
\text { UEs, since moving UEs } \\
\text { have a faster chance of } \\
\text { reconnection with } \\
\text { gNB or UE. }\end{array}$ & $\begin{array}{l}\text { Extending the LOS } \\
\text { areas of the network. } \\
\text { Putting in wireless } \\
\text { relays in order to keep } \\
\text { the LOS } \\
\text { communication and } \\
\text { optimal allocation and } \\
\text { densification of } \\
\text { heterogeneous network } \\
\text { elements composed of } \\
\text { BSs differing in size } \\
\text { and capacity. } \\
\text { Using intelligent } \\
\text { reflective surfaces. }\end{array}$ \\
\hline
\end{tabular}


Table 3. Cont

\section{Usage of}

beamforming for

transmission of

signals from

BS to UEs
Improves the coverage and signal quality by focusing the powerful signals toward a particular device.
Mismatch between the beams of the transmitter and receiver that reduce or completely eliminate the possibility of connection. Mismatched beams can cause long or short interruptions that can impact the performance of TCP.
Prevent TCP from establishing reliable end-to-end connections.

High end-to-end

throughput

degradation in the case of NLOS

communication since

SNR at the location of

UE cannot reach the expected values. The longer interruptions have a stronger impact on TCP performance due to the higher probability of triggering the RTOs, which further initialises the congestion window and slows the sending rate.
Implementation of a $5 \mathrm{G}$ core network to support:

- $\quad$ service-based architecture,

- network slicing,

- SDN/NFV concepts
Ensures data transmission between different parts of radio access networks through a core $5 \mathrm{G}$ network. Ensures the realisation of a stand-alone 5G network.
Ensuring the parallel and isolated functionality of different services. Enabling appropriate separation among the different network slices.

Implementation of user and data planes in separate SDN/NFVs.
TCP end-to-end congestion and flow control issues due to:

- the large number of simultaneously supported services,

- $\quad$ the existence of a huge number of different network slices,

- the separation of data and user plane traffic.
- Development of advanced beamforming algorithms and beam tracking concepts.

- Implementing beam sweeping techniques, which tend to establish communication pairs after beam mismatch occurs.
- $\quad$ For services with high data rates, high-speed TCP CC algorithms can be used.

- $\quad$ For delay-sensitive services, the appropriate TCP CC algorithms can be deployed.

- Separation of the control- and user-plane with the optimal selection of distinct TCPs for each one.

- Implementation of QUIC protocol for CC of multiplexed web streams in core networks.
Implementing large buffers can cause long TCP queues. Long waiting by the packets in buffers leads to bufferbloat problems and higher latencies. Implementing small buffers decreases latency but in the case of high channel variations, an increase in dropped packets can occur. Such an increase in the number of packet losses due to reduced buffer size strongly affects loss-based TCPs.
- Development of new techniques that will ensure a trade-off between performance and latency.

- Implementation of adjusted AQM techniques such as CoDel [57] and Flow Queue CoDel [84]. 
Table 3. Cont.

\begin{tabular}{|c|c|c|c|c|}
\hline $\begin{array}{l}\text { Constantly } \\
\text { transmitted signals }\end{array}$ & $\begin{array}{l}\text { Signals that enable } \\
\text { base station detection, } \\
\text { system information } \\
\text { broadcasts, channel } \\
\text { estimation, etc. }\end{array}$ & $\begin{array}{l}\text { Constantly } \\
\text { transmitted signals } \\
\text { are independent of } \\
\text { the UEs traffic. Such } \\
\text { transmission } \\
\text { consumes a part of } \\
\text { the network capacity } \\
\text { and negatively } \\
\text { impacts on the energy } \\
\text { consumption of the } \\
\text { network devices (BSs) }\end{array}$ & $\begin{array}{l}\text { Constantly transmitted } \\
\text { signals contribute to } \\
\text { the increase of } \\
\text { redundant traffic and } \\
\text { network interference. } \\
\text { This affects the TCP } \\
\text { performance in terms } \\
\text { of CC and the fair } \\
\text { distribution of data } \\
\text { flows among the users. }\end{array}$ & $\begin{array}{l}\text { Implementation of } 5 \mathrm{G} \\
\text { networks based on the } \\
\text { ultra-lean design based } \\
\text { on smart signalling } \\
\text { exchange. The } \\
\text { ultra-lean design can } \\
\text { reduce traffic and } \\
\text { congestion events, and } \\
\text { improve the TCP } \\
\text { functionality. }\end{array}$ \\
\hline $\begin{array}{l}\text { Using edge } \\
\text { computing with the } \\
\text { support of network } \\
\text { slicing }\end{array}$ & $\begin{array}{l}\text { Reduction of the } \\
\text { network latency } \\
\text { through the } \\
\text { optimised allocation } \\
\text { of computing } \\
\text { resources. }\end{array}$ & $\begin{array}{l}\text { Large delays in the } \\
5 \mathrm{G} \text { network } \\
\text { negatively impact the } \\
\text { TCP's functionality, } \\
\text { especially those } \\
\text { based on loss-based } \\
\text { TCP protocols. }\end{array}$ & $\begin{array}{l}\text { Implementing separate } \\
\text { TCP algorithms in each } \\
\text { slice to ensure optimal } \\
\text { CC. Part of the } \\
\text { applications deployed } \\
\text { in the user-plane } \\
\text { between the core and } \\
\text { access network. }\end{array}$ & $\begin{array}{l}\text { Solutions concerning } \\
\text { the allocation of the } \\
\text { servers close to UEs } \\
\text { using approaches based } \\
\text { on content delivery } \\
\text { network (CDN). } \\
\text { Development of novel } \\
\text { TCP CC algorithms } \\
\text { customised to the needs } \\
\text { of a specific } \\
\text { network slice. }\end{array}$ \\
\hline
\end{tabular}

\subsection{Usage of High Frequencies in the mmWave Spectrum}

The main benefit that the usage of high frequencies in the mmWave spectrum brings is achieving a higher throughput for a massive number of connected devices. Transmission at higher frequencies consequently leads to path loss and blockages since high frequencies cannot penetrate through obstacles (Figure 8). It is expected that due to the frequent LOS-NLOS transitions, the performance of $5 \mathrm{G}$ mmWave networks will be characterised by the occurrence of frequent blockages (Table 3). These blockages cause longer RTTs, higher packet loss probability and RTO expiration, which further degrades the TCP performance over $5 \mathrm{G}$ mmWave networks. The negative effects can be more severe for static objects compared to moving objects where there is a higher chance of a faster reconnection between a UE and a gNB.

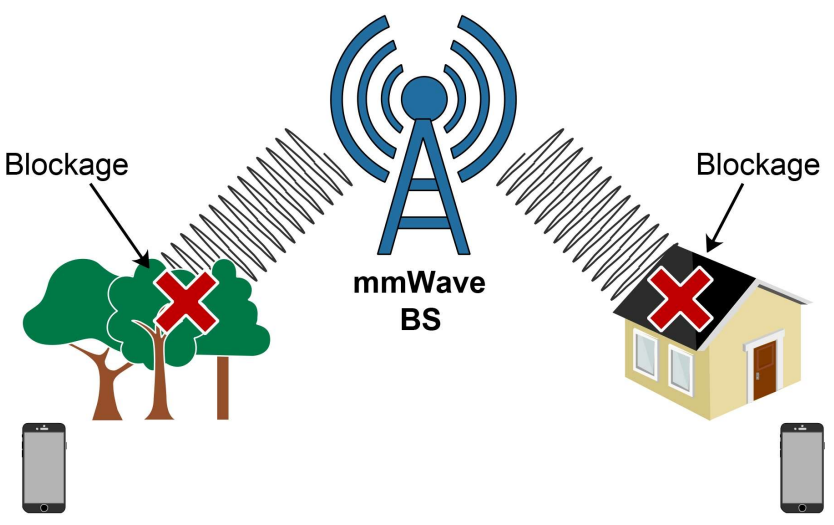

Figure 8. High-frequency millimetre waves experiencing blockages.

Although the use of the mmWave bands ensures that there is a large bandwidth and a high spectral efficiency in the channel, mmWave links suffer from high variability in terms of channel quality due to the signal losses caused by blockages. 
To evaluate the performance of the TCP CC in a highly variable mmWave channel, an end-to-end simulation framework was developed in Ref. [76]. This simulation evaluates the performance of the NewReno and CUBIC TCP CC algorithms in a varying $5 \mathrm{G}$ mmWave environment. Between the mobile user and the mmWave BS, various obstacles were placed to simulate blockage and outage events. The authors intentionally forced an RTO expiration with two outage events ( $0.4 \mathrm{~s}$ and $1 \mathrm{~s}$ long). In the short outage event, cwnd never decreased due to the lower layer retransmissions, and both NewReno and CUBIC showed a good level of performance. In the longer outage event, the RTO expired causing TCP CC to enter the slow start phase, reducing cwnd to 1 and halving the ssthresh size. Although TCP NewReno and TCP CUBIC showed latency reduction, they experienced significant throughput degradation and the slow recovery of the link capacity [76].

Furthermore, the authors in Ref. [12] analysed the performance of multiple CC algorithms using single flow blockage scenarios in a mmWave environment. Several different CC algorithms, classified as loss-based, delay-based, and hybrid, were analysed. The analysis has shown that the longer NLOS periods caused by blockages can affect the performance of the loss-based CUBIC and Scalable TCP algorithms, which repeatedly enter the slow start phase when trying to recover the sending rate. As a consequence of the LOS-NLOS transitions, the results showed the high variability of the RTT metrics. The delay-based Vegas TCP CC algorithm exhibited low RTT values but has the lowest throughput utilisation among the other CC algorithms. The hybrid-based YeAH and BBR TCP CC algorithms displayed a low queuing delay and minimal fluctuations in the RTT metrics, offering low RTT values and throughput comparable to the loss-based CC algorithms.

The solution required to mitigate the blockage problem in a mmWave band is network densification where the heterogeneous ultra-dense deployment of mmWave BSs will provide more choice of serving BSs for each UE [85] (Table 3). Deploying wireless relays can mitigate the blockage problem as they can effectively restore the communication links. Wireless relays can be based on multi-hop transmissions [86], and the development of TCP $\mathrm{CC}$ in such environments represents an important research topic. Furthermore, a solution for high penetration losses can include the use of intelligent reflecting surfaces (IRSs) that can help to bypass the obstacle creating a virtual LOS between the UE and the BS [87].

\subsection{Horizontal and Vertical Handovers}

Considering the upcoming trend characterised by the densification of $5 \mathrm{G}$ networks with BSs of different sizes and capacities, it can be expected that a lot of temporary disconnections and connections may happen in the 5G networks between UEs and BSs (Table 3). A horizontal handover is characterised by switching data sessions between different cells in UE situations exiting the specific cell or connecting to a cell that has full capacity. It is likely that this will be a frequent occurrence. Additionally, switching between different wireless technologies (e.g., from $5 \mathrm{G}$ to $4 \mathrm{G}, \mathrm{WLAN}$, etc.) is known as a vertical handover and this will also be common in the practical implementation of 5G networks (Figure 9a). 


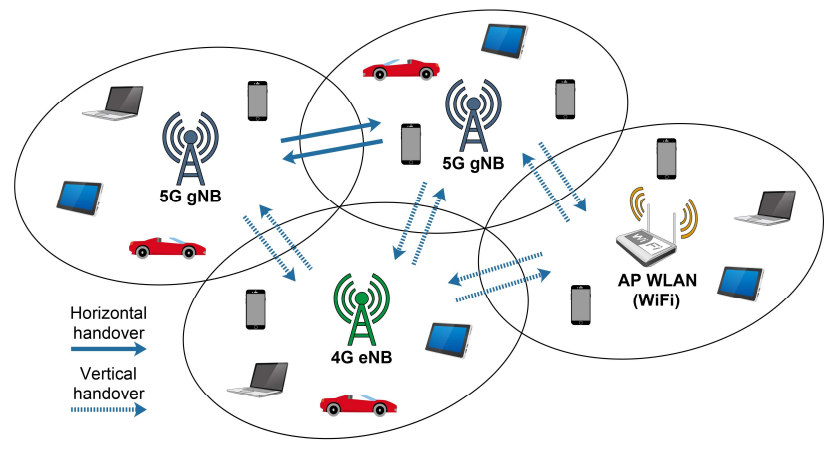

(a)

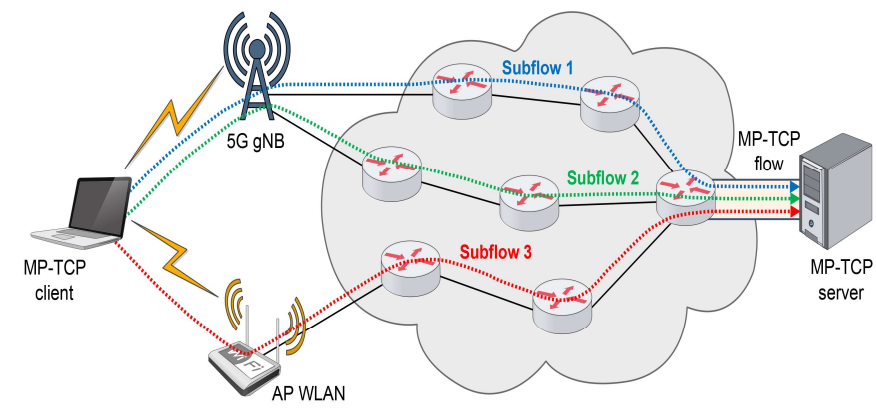

(b)

Figure 9. (a) Horizontal and vertical handovers, (b) Transfer of different MP-TCP subflows.

In addition to the aforementioned handovers, in the mmWave 5G networks, the handover can be initiated as a consequence of the high signal penetration loss that can cause blockage events. For example, when a UE connected to a cell experiences a strong degradation of signal level, it must find an adjacent cell to establish a connection. This prevents strong signal degradation and connection termination. The densification of the 5G network with a large number of BSs differing in size and capacity can efficiently alleviate the blockage problem. When a blockage event occurs in networks with an ultradense cell deployment, the UE can easily find another cell to maintain the service and connectivity using the handover technique. On the other hand, this network densification in a combination with the blockage occurrence can lead to repetitive handovers, increased handover delays and handover failures. This will cause the UEs to engage in frequent switching between adjacent cells [88] and these handovers can negatively affect the TCP performance by reducing the throughput and increasing delays (Table 3).

The handover impact on TCP performance has been analysed in Ref. [12]. The authors performed a simulation using two scenarios, a single flow with a handover and multiple concurrent flows, where the TCP CUBIC and YeAH TCP CC algorithms were compared. Since the handover in cellular networks refers to a scenario where an ongoing data session is transferred from one BS to another to ensure user connectivity while moving, in mmWave networks, besides user movement, the handover can be caused by a blockage. The single flow simulation showed that short-term blockages initiate the frequent switching of UEs between BSs, which causes degradation in the throughput. Two of the observed CC algorithms showed different recovery times in the case of rapid handover events, where CUBIC achieved an appropriate link capacity quickly while YeAH performed significantly slower. In the simulation scenario of multiple concurrent flows where multiple users are served by multiple BSs, the authors observed a negative handover impact due to the occurring blockages. Namely, when a UE moves through the environment and consequently switches between two BSs, it causes the throughput degradation of the UE that is already connected to the BS to which the moving user is connecting.

Network condition changes due to frequent blockages caused by repetitive horizontal or vertical handovers pose a real challenge in the implementation of $5 \mathrm{G}$ communications [89]. To address these challenges, different handover management schemes as presented in Refs. [88,90-92] have been considered. Moreover, the possible implementation of MP-TCP with devices containing multiple NICs and TCP flows over each NIC could represent a solution to the negative impact of frequent handovers on CC in 5G networks (Figure 9b). In the case of multiple NICs and corresponding TCP CC algorithms, the blockage of flow on one NIC will not affect flow on other NIC(s). This can contribute to uninterrupted transmission and ensured CC among communicating ends. However, devices working with multiple active NICs in parallel, will not be energy efficient. This particularly can affect sensors and battery-supplied devices. Hence, the search for the most appropriate management scheme in $5 \mathrm{G}$ mmWave networks is still an open research topic. 
Possible solutions will be dedicated to the customisation of the management schemes devised for the different services for which a specific variant of TCP is used.

\subsection{Implementation of the $5 G$ Core Network}

The 5G Core Network (5GC) specified in Ref. [93] is an evolution of the 4G LTE Core or Evolved Packet Core (EPC) architecture [94]. In contrast to EPC which includes different function modules as dedicated hardware, the 5GC architecture is designed as a servicebased architecture (SBA) provided by a set of network functions (NFs). Moreover, 5GC introduces the mechanism that includes the separation of the user plane from the control plane functions, in addition to support for network slicing (Figure 10) in different 5G usage scenarios (Figure 1) [95]. The deployment and management of different NFs in the 5G heterogeneous networks with various service requirements present a significant challenge to the practical realisation of $5 \mathrm{G}$ networks. To ensure the parallel and isolated functionality of the different services and network slices and to guarantee the separation of the user and control plane, the implementation of software-defined networking (SDN) and network function virtualisation (NFV) techniques are considered to be possible solutions [96,97].

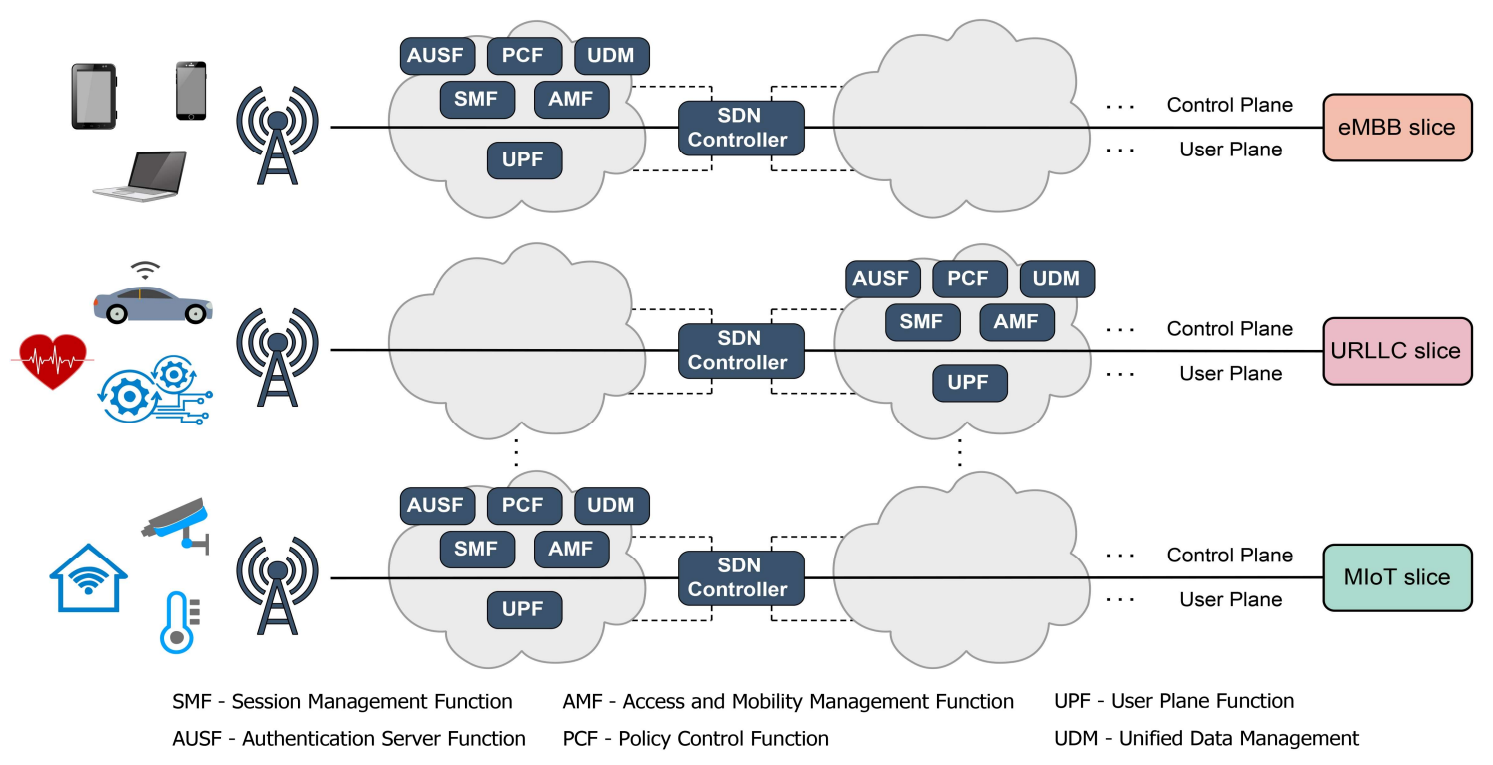

Figure 10. Control and user plane separation supported through network slicing.

However, a huge number of simultaneously supported services, the possible existence of a large number of different network slices and the separation of the data and user plane traffic can affect the performance of the TCP (Table 3). The modular design of NFs can be leveraged to deploy different $C C$ algorithms for different services, thus ensuring optimal network performance. High-speed TCPs can be used for delay-sensitive services or services requiring high data rates. The implementation of a 5GC network ensures the realisation of a standalone 5G network where the full potential of the service-based approach can be exploited by offering services based on the system requirements. Each network slice can be optimised for the required use case using an adequate TCP CC algorithm, ultimately leading to a better end-to-end user experience (Table 3). The impact of new features supported by the 5GC network on TCP performance represents an important future research topic and needs to be analysed in detail.

Since the user plane function (UPF) is responsible for the user plane operations (i.e., packet routing/switching and gateway selection), finding a high-performance UPF for fast packet processing can significantly decrease the latency [98]. This can indirectly improve the overall TCP performance. Finding optimal user plane management tech- 
niques for 5G, as presented in Ref. [99], could be another solution to further improve TCP performance.

Due to the increasing popularity of QUIC as a TCP alternative, the author in Ref. [100] has conducted a testbed experiment of QUIC performance in a 5G core network for satisfying different quality of service (QoS) criteria. The author has shown that QUIC is more resilient than TCP in a 5G core network under poor network conditions (high packet loss rate, high latency, and low bandwidth) and under optimal network conditions as well. Additionally, due to the faster connection establishment compared to TCP, the author has confirmed that QUIC can achieve significantly better performance in the 5G core network. Hence, the examination of the possible implementation of QUIC in 5G core networks represents an open research field.

\subsection{Usage of Beamforming}

For the transmission of signals from BS to UE, the use of massive antenna arrays in combination with beamforming techniques can alleviate the mmWave signal propagation issues such as high path losses (Table 3). Beamforming improves the coverage and signal quality by focusing the powerful signals toward a particular device (Figure 11). The precise alignment of directional beams in a mmWave $5 \mathrm{G}$ network requires advanced beam management algorithms. This can be ensured by the use of effective control layer procedures [101], such as initial access [102] and beam tracking [103]. However, a misalignment or mismatch between the transmitter beam and receiver beam can lead to a low receiving gain, reduced throughput or connection loss. Furthermore, forming the directional beams between gNB and the UE can compensate for a high path loss. On the other hand, it can cause a blockage problem due to the high beam directivity (Figure 11) [104]. Misalignment problems can severely degrade the TCP performance (Table 3), especially in a high mobility scenario where the beam mismatch occurs more frequently causing intense SNR fluctuations. These fluctuations can cause intermittent interruptions that can vary based on the size of obstacles and the speed of the UEs (from short to long disconnections). Although both disconnections can affect the performance of the TCP, the effects of the long ones are stronger [105]. This is due to the high probability of triggering the RTO, which leads to a congestion window initialisation and the dramatic slowing of the sending rate.

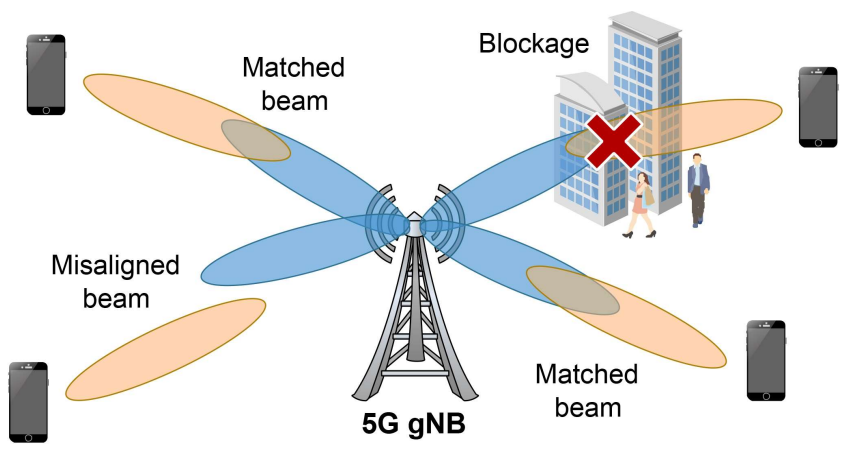

Figure 11. Beamforming scenarios in $5 \mathrm{G}$ mobile networks.

To address the impacts of beam misalignment and blockage problems on TCP performance in the mmWave frequency bands, the authors in Ref. [104] have proposed a deeplearning-based TCP (DL-TCP) algorithm. The proposed algorithm is compared against the existing NewReno, CUBIC and BBR TCP CC algorithms in a disaster 5G mmWave network where frequent blockages and beam mismatches are expected. To address the beam misalignment problem, the beam sweeping technique was used. The simulation study showed that the DL-TCP algorithm brings in improvements in performance when compared to other CC algorithms in 5G disaster environments. This is a consequence of the deep learning approach used by the DL-TCP algorithm. Such an approach can predict the link disconnection time when a packet loss occurs and can adjust the cwnd size accordingly. 
Due to the large amounts of bandwidth and high spectral efficiency, the 5G mmWave frequencies can enable multi-gigabit data rates. However, transmission at such high frequencies imposes high signal propagation losses and the usage of highly directional beams is necessary to compensate for these losses. To effectively use one of the beamforming techniques, it is necessary to overcome these issues. The solution to the beamforming issues in a challenging mmWave environment can be found in the efficient beam management techniques [101] combined with a robust beam tracking strategyand the implementation of a customised TCP CC algorithm [106]. The implementation of these combined approaches and strategies currently represent an open research topic.

\subsection{Implementation of Edge Computing}

One of the three main 5G use cases is the possibility of enabling URLLC services (e.g., remote healthcare, intelligent transportation and real-time services). The URLLC requires ultra-low latencies for critical applications characterised by ultra-high reliability and availability (Figure 1). In 5G networks operating in the mmWave bands, latency can be seriously degraded due to blockages or beam misalignment. Large delays in the $5 \mathrm{G}$ network strongly impair TCP performance (Table 3). It is crucial to reduce the end-to-end transmission latency in the mmWave 5G networks, especially when the loss-based TCP algorithms are implemented. Using TCP optimisation techniques such as the AQM can contribute to the mitigation of the RTT delay to some extent. However, for the URLLC usage scenarios, the network infrastructure needs to be adapted to meet these high requirements.

One of the emerging technologies that can address these issues is edge computing. Edge computing is developed to overcome traditional cloud computing problems (such as high latency) by bringing cloud capabilities closer to the users (Figure 12) [107]. Since the RTT value depends on the sum of the three main factors of communication, computational and propagation delay, it can be expected that the full implementation of edge computing can greatly improve the overall TCP performance. By providing storage and computational capabilities near to the location of the users, edge computing efficiently addresses the propagation delay by shortening the distance between the communication ends. Besides the possible improvement of the TCP performance, the implementation of edge computing can consequently reduce the communication delay by offering higher data rates [107].

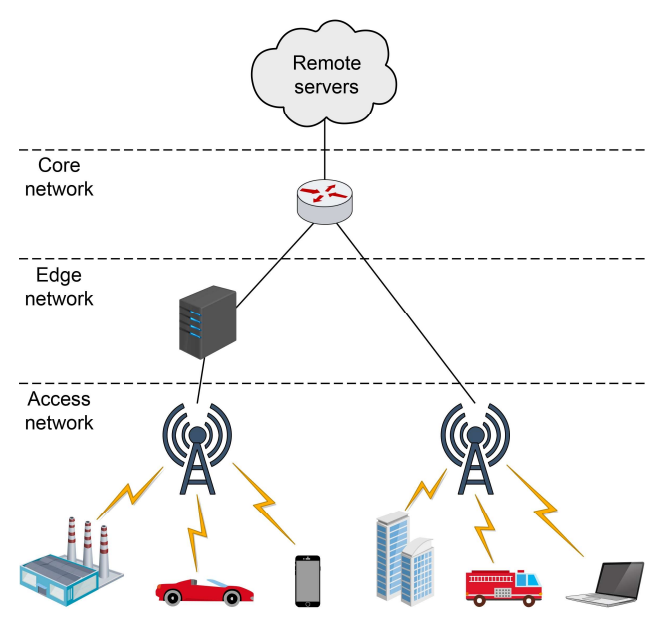

Figure 12. Edge computing model.

The impact on TCP performance using remote servers and edge server deployment has been analysed in Ref. [13] using UT high-speed movements in dense urban scenarios. For both scenarios, the authors compared several loss-based CC algorithms and the hybrid BBR algorithm. The comparison was performed for a setup involving a remotely deployed server having long RTT and a mobile edge computing (MEC) deployment [108]. The server was located in the proximity of the gNB, which ensures low latency. The results show that 
in a high-speed scenario, edge server deployment greatly reduces the RTT for all of the observed CC algorithms. In terms of the achieved goodput, edge server scenarios have a positive impact in most situations due to the presence of loss-based algorithms. However, for the BBR algorithm, the results showed a negligible improvement.

The authors have shown that, in the dense urban scenario, only the TCP BBR in the edge server deployment can satisfy the typical 5G requirements (goodput larger than $100 \mathrm{Mbps}$ and a latency lower than $10 \mathrm{~ms}$ ) operating under stable channel conditions. As presented, the usage of edge computing technology can greatly improve the data rates and decrease latency, especially for loss-based algorithms. The reason for this is due to the shorter control loop resulting from the shorter distance between the UEs and the server, which leads to a faster reaction to congestion. However, further investigations are needed in order to deduce how TCP CC in different $5 \mathrm{G}$ use cases can benefit from the implementation of edge computing technologies.

\subsection{Implementation of Buffering for Radio Link Control}

To prevent packet drops caused by high transmission link occupancy, the RLC layer performs a temporal buffering of the packets (Figure 13). The size of the RLC buffer can have a significant impact on the TCP performance (Table 3). Deploying large buffers prevents buffer overflow and reduces the chance of packet drops (Figure 13b). On the other hand, large buffers can dramatically increase latency, which can be a consequence of the long queuing delays. Such delays can cause bufferbloat problems in the mmWave $5 \mathrm{G}$ networks (Figure 13b). Reducing the buffer size can mitigate the bufferbloat problem. However, reducing the buffer size can also have a negative impact on the TCP performance (Figure 13a). More specifically, using small buffers can greatly affect the performance of the TCP CC algorithms, especially those that are loss-based, due to the high packet losses in a buffer overflow situation (Table 3). Additionally, high link variations of the mmWave channels can result in frequent NLOS communication periods. These periods can cause a faster buffer overflow what consequently leads to frequent packet drops and decreased throughput.

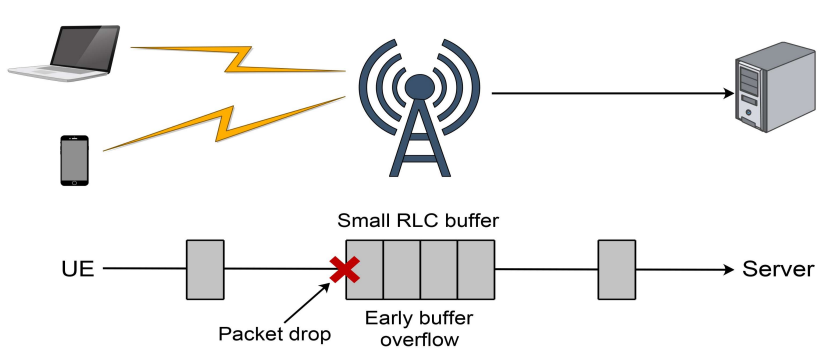

(a)

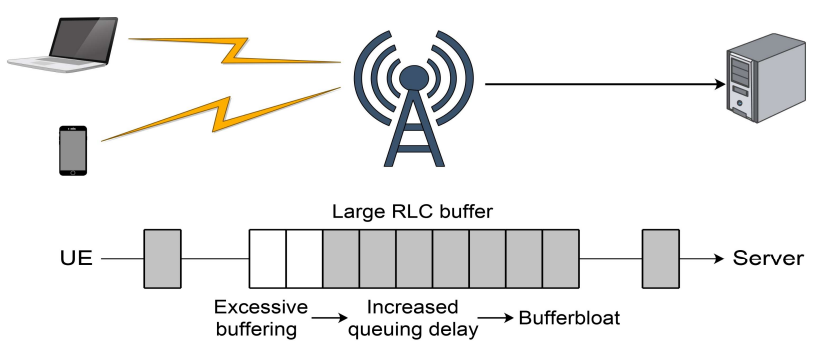

(b)

Figure 13. Different buffer size implementations: (a) packet drops due to a small RLC buffer, (b) bufferbloat problem due to a large RLC buffer.

The described issues related to the selection of the optimal buffer size in the RLC of mmWave 5G networks motivates investigations which analyse the impact of buffer size on the TCP performance. More specifically, the authors in Ref. [12] analysed the impact of different RLC buffer sizes on the TCP CC performance. The analysis showed that the frequent packet losses caused by small buffer sizes prevented loss-based algorithms such as CUBIC from reaching the congestion avoidance phase. Furthermore, the authors in Ref. [10] showed that the best results in terms of the TCP CC can be achieved when the optimal RLC buffer size is used in combination with a reduced RTO value.

Ref. [13] analysed the impact of RLC buffer size on goodput and latency. A performance comparison among the different loss-based algorithms, such as TCP NewReno, HighSpeed TCP, TCP CUBIC, and hybrid TCP BBR, was undertaken. The authors showed 
that large buffers can offer a higher goodput. This is the consequence of the higher robustness of the mmWave channel quality variations and the lower possibility of buffer overflow. Nevertheless, it is shown that using large buffers significantly increases latency. This is due to the large buffer occupancy, which can further lead to bufferbloat problems. It is also shown that deploying small buffers decreases the latency at the expense of goodput.

Additionally, the authors analysed the usage of large buffers in combination with the CoDel AQM technique. In comparison to the scenario lacking AQM technique, the obtained results show a decrease in goodput in relation to the loss-based CC algorithms. However, the decreased goodput was still larger than that obtained by the small buffer size. It is further shown that applying AQM for loss-based CC algorithms contributes to the significant latency reduction, which is comparable to the levels of latency reduction in the case of small buffer deployment. The hybrid TCP BBR algorithm in a remote server scenario using AQM showed a large decrease in latency and the same goodput as when no AQM was applied.

The authors showed that the best goodput was the hybrid TCP BBR algorithm in combination with a large buffer size. However, the obtained goodput was still under the maximum achievable rate. A downside was that the hybrid TCP BBR with a large buffer size had the highest latency compared to the other CC algorithms.

According to the presented analyses, it can be seen that an inappropriate RLC buffer size can cause buffer overflow or the bufferbloat problem. This can lead to an increased packet latency or packet drops respectively. Determining the optimal size of the RLC buffer and using different AQM techniques, such as CoDel and Flow Queue CoDel [84], can ensure a trade-off between throughput and latency. Finding solutions that will ensure an optimal buffer size in the RLC layer of mmWave 5G networks represents an open research topic. Further research attempts are needed to address this issue.

\subsection{Constantly Transmitted Signals}

Besides the user traffic signals, in mobile networks, BSs transmit signals at regular intervals used for different purposes such as BS detection, synchronisation, system information broadcasts, channel estimations etc. [109] (Table 3). The transmission of these signals is done regardless of the user traffic (Figure 14a). Although these signals will not consume a significant percentage of the overall channel usage, 5G will be based on ultra-dense cell allocation. These "always-on" signals can pose a problem in emerging 5G heterogeneous networks (Figure 14). Due to the constant nature of the transmissions, these signals can create interference between the adjacent cells, which further reduces the throughput (Table 3). The constant transmission of signals has a negative impact on the overall energy consumption of the cellular network $[110,111]$. The increased traffic in combination with the always-on signals causing increased interference can affect the TCP performance in terms of the increased number of congestion events (Table 3). This leads to a degraded throughput and raises issues related to the fairness of the distribution of the TCP flows for different users.

The main advancement in 5G mobile networks dedicated to the mitigation of negative effects of constantly transmitted signals is the implementation of an ultra-lean design in the network (Table 3). The aim of the network's ultra-lean design is to minimise the transmission of non-data signals (Figure 14b). This means that non-data signals should be transmitted only when necessary. Such an approach enables longer sleep periods for the BSs, which consequently improves the BSs energy efficiency [110]. Furthermore, the implementation of an ultra-lean design in the 5G networks will reduce the level of interference, which consequently reduces the congestion events. This can lead to improved TCP functionality (Figure 14b). However, deeper investigations are needed regarding the impact of the ultra-lean design on TCP performance. Since in the ultra-lean design non-data signals will be reduced or switched off until they are needed, TCP algorithms must be appropriately adopted to these working principles. This imposes the development of new approaches on the TCP flow control. 


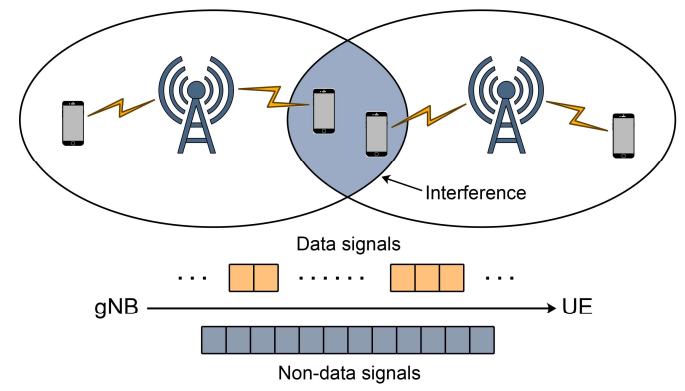

(a)

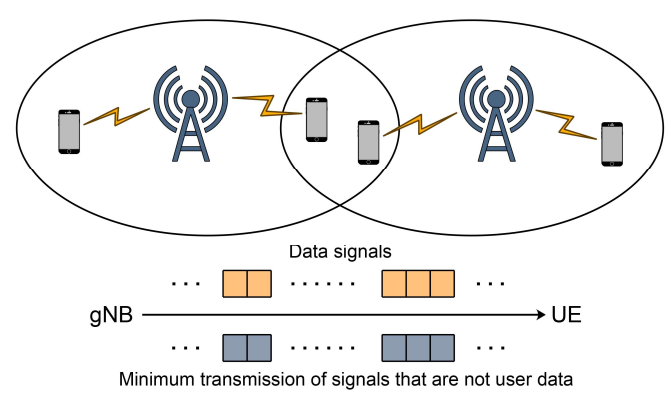

(b)

Figure 14. Activity of the radio network based on (a) always-on signals, (b) ultra-lean design.

\section{Machine Learning for Improving CC}

Today, 5G cellular networks are emerging with a large number of new use cases and applications. Conventional TCP CC algorithms that have been implemented in the last few decades are rule-based and their performance in next generation networks is often suboptimal. Conventional CC algorithms base their decisions on pre-defined criteria such as packet loss or delay and they lack the ability to learn and adapt their behaviour in complex and changing environments such as contemporary 5G cellular networks. Ref. [112] detected several constraints related to conventional CC algorithms. The main constraint is the inability to adapt to new networks where, for example, the algorithms that are designed for wired links will not perform efficiently in wireless networks. Another important constraint is an inability to learn from the previous experiences based on past information. This reduces the possibility of achieving the full potential of modern networks and results in sub-optimal performance. It is expected that using machine learning (ML) techniques will effectively overcome the limitations of the conventional TCP CC algorithms.

As opposed to conventional CC algorithms that use predefined parameters as a measure of performance effectiveness, the ML approach exploits learning techniques in order to adapt to network dynamics (Table 4). The ML CC solutions can be categorised into one of three main approaches of offline learning, online learning and deep reinforcement learning (DRL). The offline learning technique involves the definition of the network and traffic models for specific algorithms. The ML process and algorithm generation are then performed offline. To determine the optimal mappings, they are performed before the actual network implementation. The main drawback of this approach is that the emulated network and traffic model in which the algorithm is optimised may differ from the actual live network, therefore such an approach provides sub-optimal results [113]. As shown in Table 4, some of the ML CC algorithms that use offline learning are the Remy [114] and Indigo [115] ML algorithms. 
Table 4. Machine learning CC algorithms.

\begin{tabular}{|c|c|c|}
\hline Algorithm & Learning Method & Main Algorithm Characteristics \\
\hline Remy [114] & Offline & $\begin{array}{l}\text { Based on network and traffic models. Uses pre-specified } \\
\text { objectives for CC and the lookup table for maximising the } \\
\text { expected value of the objective function. }\end{array}$ \\
\hline Indigo [115] & Offline & $\begin{array}{l}\text { Congestion control oracles are generated that map the } \\
\text { algorithm state to correct actions using an emulated network } \\
\text { model. The training data is generated by applying an imitation } \\
\text { learning algorithm that uses CC oracles. }\end{array}$ \\
\hline PCC [116] & Online & $\begin{array}{l}\text { Learning is based on live experimental evidence and the utility } \\
\text { function which describes an objective. Uses multiple } \\
\text { micro-experiments to make a rate control decision and gradient } \\
\text { ascent-based online learning algorithm. }\end{array}$ \\
\hline PCC Vivace [117] & Online & $\begin{array}{c}\text { A variant of the PCC algorithm that uses a learning-theory } \\
\text { informed framework. In addition to the bandwidth and loss } \\
\text { rates, the proposed framework includes RTT gradients for } \\
\text { utility function derivations. }\end{array}$ \\
\hline Aurora [118] & DRL & $\begin{array}{l}\text { Uses a small fully connected neural network model with } \\
\text { changes in the sending rates such as agent actions. Computes } \\
\text { statistics vectors based on latency gradient, latency ratio and } \\
\text { the sending ratio. The algorithm is based on a fixed-length } \\
\text { history of the statistic vectors representing its states. The } \\
\text { algorithm gives rewards in terms of throughput improvements } \\
\text { while penalising latency and packet losses using a linear reward } \\
\text { function. The ML approach is based on the Proximal Policy } \\
\text { Optimisation (PPO) algorithm in order to train DRL agents. }\end{array}$ \\
\hline Eagle [119] & DRL & $\begin{array}{l}\text { Model training is based on a long-short term memory (LSTM) } \\
\text { neural network. The cross-entropy method is used to train a } \\
\text { DRL agent. Uses a summary of the past four observation states } \\
\text { and different reward functions for different cases. The actions of } \\
\text { the ML agent regulate the discrete changes in the sending rate } \\
\text { and the cwnd size. }\end{array}$ \\
\hline Orca [120] & DRL & $\begin{array}{c}\text { Uses DRL coarse-grain control and classic TCP CC schemes for } \\
\text { fine-grain control. This ML approach is based on the recurrent } \\
\text { neural network model. Exploits the twin delayed deep } \\
\text { deterministic policy gradient (TD3) as the training algorithm. } \\
\text { The reward function is calculated using packet delivery rate, } \\
\text { delay and loss, which averages the values to compose the } \\
\text { state space. }\end{array}$ \\
\hline
\end{tabular}

Furthermore, the online learning approach does not use fixed mappings and predefined actions. Instead, this approach uses the ML techniques in a live environment that adapt to network conditions on the fly. This approach provides a better level of performance when changing the network environment where the network parameters cannot be accurately predicted. If the network model is accurately predicted and corresponds to the actual network environment in which the algorithm is implemented, then the offline models outperform the online models [113]. The performance-oriented congestion control (PCC) [116] and the PCC Vivace [117] are examples of ML CC algorithms that use online learning techniques (Table 4).

The DRL is an ML technique that uses reinforcement learning (RL) and deep learning methods (Table 4). It is a decision-making paradigm modelled as a Markov decision process (MDP) [121]. In the framework of this approach, the DRL agent, through combining RL and deep neural networks (DNN), learns from interacting with the environment. In terms of the $\mathrm{CC}$, adjusting the cwnd size or increasing the sending rate can be viewed as the actions of the DRL agent in the decision-making process. The strategies of taking actions under the actual 
network environment are based on the policy that maximises the expected cumulative reward. The reward metric can be observed as either the throughput or network delay that is optimised for a particular system state by the actions of the DRL agent using trained DNN [112]. Some of the CC algorithms that use DRL techniques include Aurora [118], Eagle [119] and Orca [120] (Table 4).

As the ML is evolving, its application in the CC field becomes an area of particular research interest and this will become the main concept exploited in the research dedicated to the improvement of CC in 5G networks [122-124]. To the best of our knowledge, the aforementioned ML CC algorithms have not yet been evaluated using end-to-end simulations in a mmWave environment. In future research, it is expected that different ML CC algorithms will be extensively tested in various experimental $5 \mathrm{G}$ mmWave scenarios. Researches have yet to see how this cutting-edge technology will perform, especially in comparison with the existing CC algorithms in mmWave 5G networks.

\section{Research Challenges and Future Directions}

It has been shown that there is no unique TCP CC solution that can satisfy all use cases and applications, especially in a highly variable environment such as 5G mmWave networks. Developing an efficient transport layer protocol that is able to effectively utilise the mmWave bandwidth and overcome the issues in 5G network CC, such as blockages, misalignment, and handover, presents a great challenge. Future challenges are manifested in the development of an optimal solution for a particular situation. For example, in emerging usage scenarios where one device requires a high bandwidth priority and the other requires ultra-low latencies, the use of different TCP CC algorithms is expected. Scenarios in which multiple different TCP flows are controlled using different CC algorithms and compete for their fair share of the link capacity can pose a serious challenge in terms of practical realisation. More specifically, according to the presented research and the conducted simulations, the problem of fairness for different TCP flows has not yet been successfully solved. To prevent fairness issues, critical applications, such as autonomous driving or telesurgery, where ultra-high reliability and ultra-low latency are expected, should be controlled by only one TCP CC algorithm in an isolated environment.

Another challenge is to find a solution that will utilise the full channel bandwidth and minimise latency at the same time. Queuing delays as a consequence of large bottlenecks at the buffer level must be addressed as their impact can seriously deteriorate network performance. One of the solutions that is promising is the cross-layer approach where the transport layer of the Open System Interconnection (OSI) model can use the information obtained from the different layers to adjust the cwnd size accordingly.

Regardless of the method used for congestion detection, from the analysis of the presented works, we have addressed the three main challenges to TCP CC when operating in the mmWave mobile network:

- Latency vs. throughput trade-off challenge: There are various solutions to achieve low latencies. However, many of them are at the expense of bandwidth. It will be a challenge to find a solution that will achieve the best compromise for the chosen scenario.

- Queuing delay and bufferbloat problem: The problem of excess packet buffering, which creates very large queuing delays known as bufferbloat, was detected in many research studies. Nevertheless, it is a research area that still needs to be further explored in the mmWave band.

- $\quad$ Fairness problem: This occurs in non-isolated environments such as the public Internet where multiple competing TCP flows use different CC algorithms. Fairness emerges as one of the main problems and it is an area where further research is needed to find the optimal solution.

Although CUBIC is the dominant CC algorithm for the broad internet traffic today, the BBR algorithm increased its share in terms of the practical implementation and it can be expected to become the dominant algorithm in the future. As the BBR algorithm is slowly replacing the CUBIC algorithm, further research regarding their mutual interaction 
is needed to ensure the stability of the Internet. Despite the fact that BBR achieves good performance in terms of maximising the throughput and minimising latency, in highly variable mmWave environments with a massive number of connected devices, achieving an optimal network performance will be challenging. It can be concluded that in the near future, the use of BBR will be sufficient for eMBB usage scenarios, especially if it becomes the dominant algorithm. However, further research is needed to implement TCP CC in the upcoming Internet of Everything (IoE) concept characterised by a large number of installed sensors. This will use 5G mobile networks as an infrastructural backbone.

Academic and industry researchers are constantly making efforts to improve traditional rule-based algorithms that use predefined heuristics to address new requirements. On the other hand, ML TCP CC is in its early stages and it remains to be seen whether the future will be consistent with the traditional CC paradigm or if the future of TCP CC lies in intelligent ML algorithms. The deployment of the ML in TCP CC is still in its infancy and it is too early to expect any significant application at a higher level. However, with the advent of 6G networks, ML will need to be considered as the dominant direction in the field of network CC. There are possible directions in the implementation of the ML for CC. The first one is based on the development of entirely new ML-based algorithms that will completely replace the existing ones. The second is based on the integration of some of the ML properties into the existing algorithms and maintaining its backward compatibility with rule-based algorithms. The future will show which of these directions will dominate.

\section{Conclusions}

The 5G mobile networks must accommodate high demands and support various use cases. Besides sub- $6 \mathrm{GHz}$ frequency bands, the new mmWave spectrum is considered in the 5G mobile communications due to the high spectral efficiency, high spatial reuse, low latencies, and multi-gigabit data rates. However, the implementation of the 5G network in sub-6 GHz and mmWave frequency bands brings new challenges in the realisation of TCP CC.

In this paper, a comprehensive survey of TCP algorithms used for CC is presented. An overview of surveyed algorithms includes single-flow, multi-flow TCP CC algorithms, and alterative algorithms that have been envisioned as the future replacement for TCP. TCP and the concept of CC have been presented through an overview of the relevant related works that are primarily focused on the current cognitions concerning the challenges in the improvement of CC functionality in the 5G networks. The CC implementation challenges in the $5 \mathrm{G}$ networks can be caused by blockage problems, beam misalignment, frequent handovers, inadequate buffer sizes, interference due to the constant transmission of non-data signals, and changes in the data flow due to the usage of edge computing. These challenges can degrade the ТСР CC performance and, in this work, each challenge has been explained. The current research attempts for alleviating the issues related to every challenge have been elaborated on. Due to the challenges and limitations that conventional CC algorithms involve, it is realistic to expect further advancements of the TCP CC algorithms in the years to come.

These advancements are also expected in the implementation of the ML techniques, which been overviewed in terms of improving CC in the 5G networks. Therefore, the most relevant smart ML-based CC algorithms with the ability to learn and adapt to future complex networks are overviewed. Finally, a discussion related to the main challenges that must be addressed for the efficient implementation of CC algorithms in the upcoming 5G mobile networks has been presented. The discussion emphasises the potential directions in the development of ML-based algorithms as the most promising candidates for the implementation of CC in complex 5G and future $6 \mathrm{G}$ networks.

Author Contributions: Conceptualization, J.L.; methodology, Z.K.; validation, J.L. and J.O.; formal analysis, J.L. and Z.K.; investigation, Z.K.; writing-original draft preparation, J.L. and Z.K.; writingreview and editing, J.L.; visualization, J.L. and Z.K.; supervision, J.L. and J.O. All authors have read and agreed to the published version of the manuscript. 
Funding: This research received no external funding.

Institutional Review Board Statement: Not applicable.

Conflicts of Interest: The authors declare no conflict of interest.

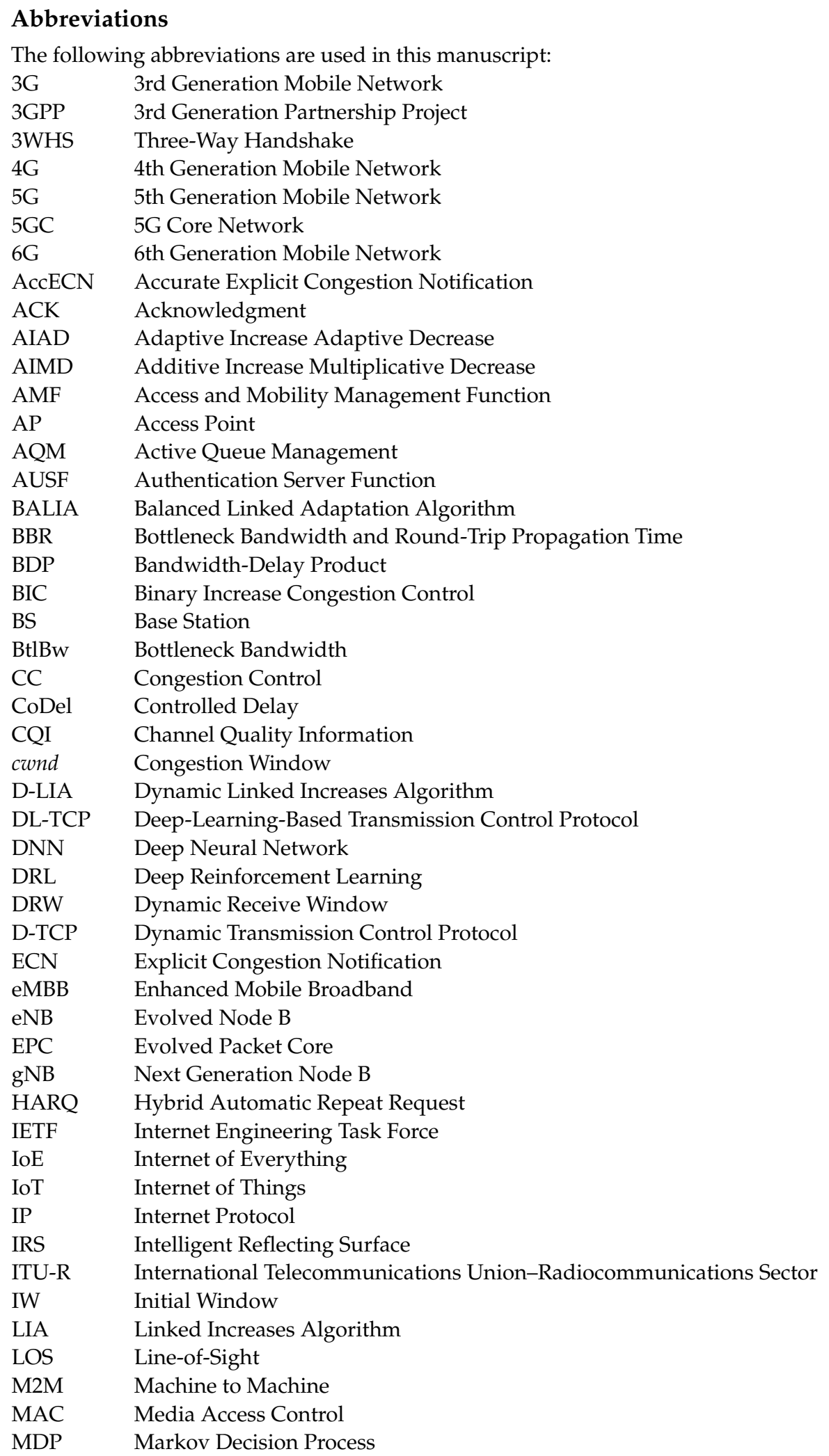




\begin{tabular}{|c|c|}
\hline MEC & Mobile Edge Computing \\
\hline MIoT & Massive Internet of Things \\
\hline ML & Machine Learning \\
\hline mMIMO & Massive Multiple-Input and Multiple-Output \\
\hline mMTC & Massive Machine-Type Communication \\
\hline mmWave & Millimeter-Wave \\
\hline MP-TCP & Multipath TCP \\
\hline MP-QUIC & Multipath QUIC \\
\hline $\mathrm{ms}$ & millisecond \\
\hline MSS & Maximum Segment Size \\
\hline NF & Network Function \\
\hline NFV & Network Function Virtualization \\
\hline NIC & Network Interface Card \\
\hline NLOS & Non-Line-of-Sight \\
\hline OLIA & Opportunistic Linked Increases Algorithm \\
\hline OSI & Open System Interconnection \\
\hline PCC & Performance-Oriented Congestion Control \\
\hline PCF & Policy Control Function \\
\hline QoS & Quality of Service \\
\hline QUIC & Quick User Datagram Protocol Internet Connection \\
\hline RL & Reinforcement Learning \\
\hline RLC & Radio Link Control \\
\hline RTO & Retransmission Time-Out \\
\hline RtProp & Round Trip Propagation Time \\
\hline RTT & Round-Trip-Time \\
\hline RTW-TCP & Real-Time Wireless Transmission Control Protocol \\
\hline rwnd & Receiver Window \\
\hline SA & Standalone \\
\hline SACK & Selective Acknowledgment \\
\hline SBA & Service-Based Architecture \\
\hline SINR & Signal to Interference and Noise Ratio \\
\hline SMF & Session Management Function \\
\hline ssthresh & Slow-Start Threshold \\
\hline SYN & Synchronize \\
\hline TCP & Transmission Control Protocol \\
\hline TFO & Transmission Control Protocol Fast Open \\
\hline TLS & Transport Layer Security \\
\hline TMRG & Transport Modeling Research Group \\
\hline UDM & Unified Data Management \\
\hline UDP & User Datagram Protocol \\
\hline UE & User Equipment \\
\hline URLLC & Ultra-Reliable and Low Latency Communication \\
\hline WiFi & Wireless Fidelity \\
\hline WLAN & Wireless Local Area Network \\
\hline YEAH & Yet Another Highspeed Transmission Control Protocc \\
\hline
\end{tabular}

\section{References}

1. IMT Vision-Framework and Overall Objectives of the Future Development of IMT for 2020 and Beyond. ITU-R Recommendation M.2083-0. September 2015. Available online: https:/ / www.itu.int/dms_pubrec/itu-r/rec/m/R-REC-M.2083-0-201509-I!!PDF-E. pdf (accessed on 31 April 2021).

2. Jejdling, F. Ericsson Mobility Report. November 2020. Available online: https://www.ericsson.com/4adc87/assets/local/ mobility-report/documents/2020/november-2020-ericsson-mobility-report.pdf (accessed on 31 April 2021).

3. Minimum Requirements Related to Technical Performance for IMT-2020 Radio Interface(s). ITU-R Report M.2410-0. November 2017. Available online: https://www.itu.int/dms_pub/itu-r/opb/rep/R-REP-M.2410-2017-PDF-E.pdf (accessed on 31 April 2021).

4. Chen, H.; Abbas, R.; Cheng, P.; Shirvanimoghaddam, M.; Hardjawana, W.; Bao, W.; Li, Y.; Vucetic, B. Ultra-Reliable Low Latency Cellular Networks: Use Cases, Challenges and Approaches. IEEE Commun. Mag. 2018, 56, 119-125. [CrossRef] 
5. Understanding mmWave Spectrum for 5G Networks. 5G Americas White Paper. December 2020. Available online: https: //www.5gamericas.org/wp-content/uploads/2020/12/InDesign-Understanding-mmWave-for-5G-Networks.pdf (accessed on 31 April 2021).

6. Pi, Z.; Khan, F. An introduction to millimeter-wave mobile broadband systems. IEEE Commun. Mag. 2011, 49, 101-107. [CrossRef]

7. GSMA. 5G Spectrum GSMA Public Policy Position. March 2021. Available online: https://www.gsma.com/spectrum/wpcontent/uploads/2021/04/5G-Spectrum-Positions.pdf (accessed on 31 April 2021).

8. Brenner, D. Global 5G Spectrum Update. June 2020. Available online: https://www.qualcomm.com/media/documents/files/ 5g-spectrum-update-for-mipi-alliance.pdf (accessed on 31 April 2021).

9. Yang, G. Throughput and Latency of Millimeter-Wave Networks: Performance Analyses and Design Principles. Ph.D Thesis, KTH Royal Institute of Technology, Stockholm, Sweeden, 2018. Available online: https:/ / kth.diva-portal.org/smash/get/diva2: 1195862/FULLTEXT02.pdf (accessed on 31 April 2021).

10. Al-Ogaili, F.; Shubair, R.M. Millimeter-wave mobile communications for 5G: Challenges and opportunities. In Proceedings of the 2016 IEEE International Symposium on Antennas and Propagation (APSURSI), Fajardo, PR, USA, 26 June-1 July 2016; pp. 1003-1004.

11. Niu, Y.; Li, Y.; Jin, D.; Su, L.; Vasilakos, A.V. A survey of millimeter wave communications (mmWave) for 5G: Opportunities and challenges. Wirel. Netw. 2015, 21, 2657-2676. [CrossRef]

12. Mateo, P.J.; Fiandrino, C.; Widmer, J. Analysis of TCP Performance in 5G mm-Wave Mobile Networks. In Proceedings of the ICC 2019-2019 IEEE International Conference on Communications (ICC), Shanghai, China, 20-24 May 2019; pp. 1-7.

13. Zhang, M.; Polese, M.; Mezzavilla, M.; Zhu, J.; Rangan, S.; Panwar, S.; Zorzi, A.M. Will TCP Work in mmWave 5G Cellular Networks? IEEE Commun. Mag. 2019, 57, 65-71. [CrossRef]

14. Allman, M.; Paxson, V.; Blanton, E. TCP Congestion Control. RFC 5681. September 2009. Available online: https:/ /tools.ietf.org/ $\mathrm{html} / \mathrm{rfc5681}$ (accessed on 31 April 2021).

15. Poorzare, R.; Aug, A.C. Challenges on the Way of Implementing TCP Over 5G Network. IEEE Access 2020, 8, 176393176415. [CrossRef]

16. Cerf, V.G.; Kahn, R.E. A Protocol for Packet Network Intercommunication. IEEE Trans. Commun. 1974, 22, 637-648. [CrossRef]

17. Postel, J. Transmission Control Protocol. RFC 793. September 1981. Available online: https://tools.ietf.org/html/rfc793 (accessed on 31 April 2021).

18. Cheng, Y.; Chu, J.; Radhakrishnan, S.; Jain, A. TCP Fast Open. RFC 7413. December 2014. Available online: https:/ / datatracker. ietf.org/doc/html/rfc7413 (accessed on 31 April 2021).

19. Clark, D.D. Window and Acknowledgement Strategy in TCP. RFC 813. July 1982. Available online: https://tools.ietf.org/html/ rfc813 (accessed on 31 April 2021).

20. Turkovic, B.; Kuipers, F.A.; Uhlig, S. Fifty Shades of Congestion Control: A Performance and Interactions Evaluation. arXiv 2019, arXiv:1903.03852.

21. Gettys, J.; Nichols, K. Bufferbloat: Dark Buffers in the Internet: Networks without effective AQM may again be vulnerable to congestion collapse. Queue 2011, 9, 40-54. [CrossRef]

22. Jacobson, V. Congestion Avoidance and Control. Comput. Commun. Rev. 1988, 18, 314-329. [CrossRef]

23. Chu, J.; Dukkipati, N.; Cheng, Y.; Mathis, M. Increasing TCP's Initial Window. RFC 6928. April 2013. Available online: https://tools.ietf.org/html/rfc6928 (accessed on 31 April 2021).

24. Stevens, W. TCP Slow Start, Congestion Avoidance, Fast Retransmit, and Fast Recovery Algorithms. RFC 2001. January 1997. Available online: https:/ / tools.ietf.org/html/rfc2001 (accessed on 31 April 2021).

25. Afanasyev, A.; Tilley, N.; Reiher, P.; Kleinrock, L. Host-to-Host Congestion Control for TCP. IEEE Commun. Surv. Tutor. 2010, 12, 304-342. [CrossRef]

26. Floyd, S. Metrics for the Evaluation of Congestion Control Mechanisms. RFC 5166. March 2008. Available online: https: //tools.ietf.org/html/rfc5166 (accessed on 31 April 2021).

27. Jacobson, V. Modified TCP Congestion Avoidance Algorithm; Technical Report. 1990. Available online: ftp://ftp.ee.lbl.gov/ email/vanj.90apr30.txt (accessed on 31 April 2021).

28. Fall, K.; Floyd, S. Simulation-Based Comparisons of Tahoe, Reno and SACK TCP. ACM SIGCOMM Comput. Commun. Rev. 1996, 26, 5-21. [CrossRef]

29. Henderson, T.; Floyd, S.; Gurtov, A.; Nishida, Y. The NewReno Modification to TCP's Fast Recovery Algorithm. RFC 6582. April 2012. Available online: https://tools.ietf.org/html/rfc6582 (accessed on 31 April 2021).

30. Mathis, M.; Mahdavi, J.; Floyd, S.; Romanow, A. TCP Selective Acknowledgment Options. RFC 2018. October 1996. Available online: https: / tools.ietf.org/html/rfc2018 (accessed on 31 April 2021).

31. Mascolo, S.; Casetti, C.; Gerla, M.; Sanadidi, M.Y.; Wang, R. TCP westwood: Bandwidth estimation for enhanced transport over wireless links. In Proceedings of the 7th Annual International Conference on Mobile Computing and Networking (MobiCom’01), Rome Italy, 16-21 July 2001; pp. 287-297.

32. Floyd, S. HighSpeed TCP for Large Congestion Windows. RFC 3649. December 2003. Available online: https://tools.ietf.org/ $\mathrm{html} / \mathrm{rfc} 3649$ (accessed on 31 April 2021).

33. Kelly, T. Scalable TCP: Improving Performance in Highspeed Wide Area Networks. ACM SIGCOMM Comput. Commun. Rev. 2003, 32, 83-91. [CrossRef] 
34. Xu, L.; Harfoush, K.; Rhee, I. Binary increase congestion control (BIC) for fast long-distance networks. In Proceedings of the IEEE INFOCOM, Hong Kong, China, 7-11 March 2004.

35. Ha, S.; Rhee, I.; Xu, L. CUBIC: A New TCP-Friendly High-Speed TCP Variant. ACM SIGOPS Oper. Syst. Rev. 2008, 42, 64-74. [CrossRef]

36. Brakmo, L.S.; O'Malley, S.W.; Peterson, L.L. TCP Vegas: New techniques for congestion detection and avoidance. ACM SIGCOMM Comput. Commun. Rev. 1994, 24, 24-35. [CrossRef]

37. Hasegawa, G.; Kurata, K.; Murata, A. Analysis and Improvement of Fairness between TCP Reno and Vegas for Deployment of TCP Vegas to the Internet. In Proceedings of the 2000 International Conference on Network Protocols, Osaka, Japan, 14-17 November 2000; pp. 177-186.

38. Sing, J.; Soh, B. TCP New Vegas: Improving the Performance of TCP Vegas over High Latency Links. In Proceedings of the Fourth IEEE International Symposium on Network Computing and Applications, Cambridge, MA, USA, 27-29 July 2005 ; pp. 73-82.

39. Srijith, K.; Jacob, L.; Ananda, A. TCP Vegas-A: Improving the Performance of TCP Vegas. Comput. Commun. 2005, 28, 429-440. [CrossRef]

40. Zhou, W.; Xing, W.; Wang, Y.; Zhang, J. TCP Vegas-V: Improving the performance of TCP Vegas. In Proceedings of the International Conference on Automatic Control and Artificial Intelligence (ACAI 2012), Xiamen, China, 3-5 March 2012; pp. $2034-2039$.

41. Wei, D.X.; Jin, C.; Low, S.H.; Hegde, S. FAST TCP: Motivation, Architecture, Algorithms, Performance. IEEE/ACM Trans. Netw. 2006, 14, 1246-1259.

42. Tan, K.; Song, J.; Zhang, Q.; Sridharan, M. A Compound TCP Approach for High-speed and Long Distance Networks. In Proceedings of the IEEE INFOCOM 2006. 25TH IEEE International Conference on Computer Communications, Barcelona, Spain, 23-29 April 2006; pp. 1-12.

43. Baiocchi, A.; Castellani, A.P.; Vacirca, F. Yeah-tcp: Yet another highspeed TCP. In Proceedings of the 5th International Workshop on Protocols for Fast Long-Distance Networks (PFLDnet), Los Angeles, CA, USA, 7-9 February 2007.

44. Cardwell, N.; Cheng, Y.; Gunn, C.S.; Yeganeh, S.H.; Jacobson, V. BBR: Congestion-based congestion control. Commun. ACM 2017, 60, 58-66. [CrossRef]

45. Cardwell, N.; Cheng, Y.; Yeganeh, S.H.; Swett, I.; Vasiliev, V.; Jha, P.; Seung, Y.; Mathis, M.; Jacobson, V. Bbrv2: A model-based congestion control. In Proceedings of the IETF 102th Meeting, Montreal, QC, Canada, 14-20 July 2018; pp. 1-36.

46. Mishra, A.; Sun, X.; Jain, A.; Pande, S.; Joshi, R.; Leong, B. The Great Internet TCP Congestion Control Census. Proc. ACM Meas. Anal. Comput. Syst. 2019, 3, 1-24. [CrossRef]

47. Jin, C.; Wei, D.; Low, S.H.; Bunn, J.; Choe, H.D.; Doylle, J.C.; Newman, H.; Ravot, S.; Singh, S.; Paganini, F.; et al. FAST TCP: From theory to experiments. IEEE Netw. 2005, 19, 4-11.

48. Kleinrock, L. Power and Deterministic Rules of Thumb for Probabilistic Problems in Computer Communications. In Proceedings of the International Conference on Communications (ICC '79), Berlin, Germany, 17-20 September 1979; Volume 3, pp. 43.1.1-43.1.10.

49. Scholz, D.; Jaeger, B.; Schwaighofer, L.; Raumer, D.; Geyer, F.; Carle, G. Towards a Deeper Understanding of TCP BBR Congestion Control. Available online: https://www.net.in.tum.de/fileadmin/bibtex/publications/papers/IFIP-Networking-2018-TCPBBR.pdf (accessed on 31 April 2021).

50. Hock, M.; Bless, R.; Zitterbart, M. Experimental Evaluation of BBR Congestion Control. In Proceedings of the IEEE 25th International Conference on Network Protocols (ICNP), Toronto, ON, Canada, 10-13 October 2017.

51. Floyd, S. Congestion Control Principles. RFC 2914. September 2000. Available online: https://datatracker.ietf.org/doc/html/ rfc2914 (accessed on 31 April 2021).

52. Mo, J.; La, R.J.; Anantharam, V.; Walrand, J. Analysis and comparison of TCP Reno and Vegas. In Proceedings of the IEEE INFOCOM'99, Conference on Computer Communications, Eighteenth Annual Joint Conference of the IEEE Computer and Communications Societies, New York, NY, USA, 21-25 March 1999.

53. Pieska, M.; Kassler, A.J.; Lundqvist, H.; Cai, T. Improving TCP Fairness over Latency Controlled 5G mmWave Communication Links. WSA 2018. In Proceedings of the 22nd International ITG Workshop on Smart Antennas, Bochum, Deutschland, 14-16 March 2021.

54. Gong, Y.; Rossi, D.; Testa, C.; Valenti, S.; Täht, M.D. Fighting the bufferbloat: On the coexistence of AQM and low priority congestion control. In Proceedings of the 2013 IEEE Conference on Computer Communications Workshops (INFOCOM WKSHPS), Turin, Italy, 14-19 April 2013.

55. Gomez, C.A.; Wang, X.; Shami, A. Intelligent Active Queue Management Using Explicit Congestion Notification. In Proceedings of the 2019 IEEE Global Communications Conference (GLOBECOM), Waikoloa, HI, USA, 28-30 October 2019.

56. Floyd, S.; Jacobson, V. Random early detection gateways for congestion avoidance. IEEE/ACM Trans. Netw. 1993, 1, 397-413. [CrossRef]

57. Nichols, K.; Jacobson, V.; McGregor, A.; Iyengar, J. Controlled Delay Active Queue Management. RFC 8289. January 2018. Available online: https://tools.ietf.org/html/rfc8289 (accessed on 31 April 2021).

58. Al-Saadi, R.; Armitage, G.; But, J.; Branch, P. A Survey of Delay-Based and Hybrid TCP Congestion Control Algorithms. IEEE Commun. Surv. Tutor. 2019, 21,3609-3638. [CrossRef]

59. Fairhurst, G.; Welzl, M. The Benefits of Using Explicit Congestion Notification (ECN). RFC 8087. March 2017. Available online: https:/ / tools.ietf.org/html/rfc8087 (accessed on 31 April 2021). 
60. Baker, F.; Fairhurst, G. IETF Recommendations Regarding Active Queue Management. RFC 7567. July 2015. Available online: https:/ / datatracker.ietf.org/doc/html/rfc7567\#section-2.2 (accessed on 31 April 2021).

61. Briscoe, B.; Kuehlewind, M.; Scheffenegger, R. More Accurate ECN Feedback in TCP. IETF, Internet-Draft. February 2021. Available online: https:// datatracker.ietf.org/doc/html/draft-ietf-tcpm-accurate-ecn (accessed on 31 April 2021).

62. Ford, A.; Raiciu, C.; Handley, M.; Bonaventure, O.; Paasch, C. TCP Extensions for Multipath Operation with Multiple Addresses. RFC 8684. March 2020. Available online: https:/ / datatracker.ietf.org/doc/html/rfc8684 (accessed on 31 April 2021).

63. Raiciu, C.; Handly, M.; Wischik, D. Coupled Congestion Control for Multipath Transport Protocols. RFC 6356. October 2011. Available online: https:/ / datatracker.ietf.org/doc/html/rfc6356\#section-3 (accessed on 31 April 2021).

64. Khalili, R.; Gast, N.; Popovic, M.; Boudec, J.L. MPTCP Is Not Pareto-Optimal: Performance Issues and a Possible Solution. IEEE/ACM Trans. Netw. 2013, 21, 1651-1665. [CrossRef]

65. Peng, Q.; Walid, A.; Hwang, J.; Low, S.H. Multipath TCP: Analysis, Design, and Implementation. IEEE/ACM Trans. Netw. 2016, 24, 596-609. [CrossRef]

66. Polese, M.; Jana, R.; Zorzi, M. TCP in 5G mmWave networks: Link level retransmissions and MP-TCP. In Proceedings of the 2017 IEEE Conference on Computer Communications Workshops (INFOCOM WKSHPS), Atlanta, GA, USA, 1 May 2017.

67. Fu, F.; Zhou, X.; Dreibholz, T.; Wang, K.; Zhou, F.; Gan, Q. Performance comparison of congestion control strategies for multi-path TCP in the NORNET testbed. In Proceedings of the 2015 IEEE/CIC International Conference on Communications in China (ICCC), Shenzhen, China, 2-4 November 2015.

68. Lubna, T.; Mahmud, I.; Cho, Y.-Z. D-LIA: Dynamic congestion control algorithm for MPTCP. ICT Express 2020, 6, 263-268. [CrossRef]

69. Iyengar, J.; Thomson, M. QUIC: A UDP-Based Multiplexed and Secure Transport. RFC 9000. May 2021. Available online: https:/ / datatracker.ietf.org/doc/html/rfc9000 (accessed on 31 April 2021).

70. Bishop, M. Hypertext Transfer Protocol Version 3 (HTTP/3). IETF, Internet-Draft. February 2021. Available online: https: / / datatracker.ietf.org/doc/html/draft-ietf-quic-http-34 (accessed on 31 April 2021).

71. Iyengar, J.; Swett, I. QUIC Loss Detection and Congestion Control. RFC 9002. May 2021. Available online: https:// datatracker. ietf.org/doc/html/rfc9002 (accessed on 31 April 2021).

72. Cook, S.; Mathieu, B.; Truong, P.; Hamchaoui, I. QUIC: Better for what and for whom? In Proceedings of the 2017 IEEE International Conference on Communications (ICC), Paris, France, 21-25 May 2017.

73. Yu, Y.; Xu, M.; Yang, Y. When QUIC meets TCP: An experimental study. In Proceedings of the 2017 IEEE 36th International Performance Computing and Communications Conference (IPCCC), San Diego, CA, USA, 10-12 December 2017.

74. Coninck, Q.D.; Bonaventure, O. Multipath QUIC: Design and Evaluation. In Proceedings of the 13th International Conference on Emerging Networking EXperiments and Technologies (CoNEXT '17), Incheon, Korea, 12-15 December 2017.

75. Coninck, Q.D.; Bonaventure, O. Multipath Extensions for QUIC (MP-QUIC). IETF Internet-Draft. May 2021. Available online: https://datatracker.ietf.org/doc/draft-deconinck-quic-multipath/07/ (accessed on 31 April 2021).

76. Zhang, M.; Mezzavilla, M.; Ford, R.; Rangan, S.; Panwar, S.; Mellios, E.; Kong, D.; Nix, A.; Zorzi, M. Transport layer performance in 5G mmWave cellular. In Proceedings of the 2016 IEEE Conference on Computer Communications Workshops (INFOCOM WKSHPS), San Francisco, CA, USA, 10-14 April 2016.

77. Mezzavilla, M.; Zhang, M.; Polese, M.; Ford, R.; Dutta, S.; Rangan, S.; Zorzi, M. End-to-end simulation of 5G mmWave networks. IEEE Commun. Surv. Tuts. 2018, 20, 2237-2263. [CrossRef]

78. Zhang, M.; Mezzavilla, M.; Zhu, J.; Rangan, S.; Panwar, I.S. TCP dynamics over mmwave links. In Proceedings of the 2017 IEEE 18th International Workshop on Signal Processing Advances in Wireless Communications (SPAWC), Sapporo, Japan, 3-6 July 2017.

79. Na, W.; Lakew, D.S.; Lee, J.; Cho, S. Congestion control vs. link failure: TCP behavior in mmWave connected vehicular networks. Future Gener. Comput. Syst. 2019, 101, 1213-1222. [CrossRef]

80. Srivastava, A.; Fund, F.; Panwar, S.S. An Experimental Evaluation of Low Latency Congestion Control for mmWave Links. In Proceedings of the IEEE INFOCOM 2020-IEEE Conference on Computer Communications Workshops (INFOCOM WKSHPS), Toronto, ON, Canada, 6-9 July 2020.

81. Kanagarathinam, M.R.; Singh, S.; Sandeep, I.; Kim, H.; Maheshwari, M.K.; Hwang, J.; Roy, A.; Saxena, N. NexGen D-TCP: Next Generation Dynamic TCP Congestion Control Algorithm. IEEE Access 2020, 8, 164482-164496. [CrossRef]

82. Ricci, R.; Eide, E.; CL Team. Introducing CloudLab: Scientific infrastructure for advancing cloud architectures and applications. Mag. USENIX SAGE 2014, 39, 36-38.

83. Briscoe, B.; Schepper, K.D.; Bagnulo, M.; White, G. Low Latency, Low Loss, Scalable Throughput (L4S) Internet Service: Architecture. IETF, Internet-Draft. November 2020. Available online: https://tools.ietf.org/html/draft-ietf-tsvwg-14s-arch-08 (accessed on 31 April 2021).

84. Hoeiland-Joergensen, T.; McKenney, P.; Taht, D.; Gettys, J.; Dumazet, E. The Flow Queue CoDel Packet Scheduler and Active Queue Management Algorithm. RFC 8290. January 2018. Available online: https://tools.ietf.org/html/rfc8290 (accessed on 31 April 2021).

85. Feng, W.; Wang, Y.; Lin, D.; Ge, N.; Lu, J.; Li, S. When mmWave Communications Meet Network Densification: A Scalable Interference Coordination Perspective. IEEE J. Sel. Areas Commun. 2017, 35, 1459-1471. [CrossRef] 
86. Lorincz, J.; Ukic, N.; Begusic, D. Throughput Comparison of AODV-UU and DSR-UU Protocol Implementations in Multi-hop Static Environments. In Proceedings of the International Conference on Telecommunications Telecommunications (ConTEL 2007), Zagreb, Croatia, 13-15 June 2007.

87. Wu, Q.; Zhang, R. Towards Smart and Reconfigurable Environment: Intelligent Reflecting Surface Aided Wireless Network. IEEE Commun. Mag. 2020, 58, 106-112. [CrossRef]

88. Bilen, B.C.T.; Chowdhury, K.R. Handover Management in Software-Defined Ultra-Dense 5G Networks. IEEE Netw. 2017, 31, 49-55. [CrossRef]

89. Narayanan, A.; Ramadan, E.; Carpenter, J.; Liu, Q.; Liu, Y.; Qian, F.; Zhang, Z.-L. A First Look at Commercial 5G Performance on Smartphones. In Proceedings of the Web Conference 2020 (WWW '20), Taipei, Taiwan, 20-24 April 2020.

90. Mezzavilla, M.; Goyal, S.; Panwar, S.; Rangan, S.; Zorzi, M. An MDP model for optimal handover decisions in mmWave cellular networks. In Proceedings of the 2016 European Conference on Networks and Communications (EuCNC), Athens, Greece, 27-30 June 2021.

91. In Proceedings of the Improved Handover through Dual Connectivity in 5G mmWave Mobile Networks. IEEE J. Sel. Areas Commun. 2017, 35, 2069-2084. [CrossRef]

92. Alnabhan, M.; Al-qatawneh, E.; Alabadleh, A.; Atoum, M.; Alnawyseh, M. Efficient Handover Approach in 5G Mobile Networks. Int. J. Adv. Sci. Eng. Inform. Technol. 2020, 10, 1417-1422. [CrossRef]

93. ETSI TS 123501 V16.6.0 (2020-10), System Architecture for the 5G System (5GS) (3GPP TS 23.501 version 16.6.0 Release 16). 2020. Available online: https://www.etsi.org/deliver/etsi_ts /123500_123599/123501/16.06.00_60/ts_123501v160600p.pdf (accessed on 31 April 2021).

94. ETSI TS 123401 V8.14.0 (2011-06), General Packet Radio Service (GPRS) Enhancements for Evolved Universal Terrestrial Radio Access Network (E-UTRAN) Access (3GPP TS 23.401 version 8.14.0 Release 8). 2011. Available online: https: / / www.etsi.org/ deliver/etsi_ts/123400_123499/123401/08.14.00_60/ts_123401v081400p.pdf (accessed on 31 April 2021).

95. Ordonez-Lucena, J.; Ameigeiras, P.; Lopez, D.; Ramos-Munoz, J.J.; Lorca, J.; Folgueira, J. Network Slicing for 5G with SDN/NFV: Concepts, Architectures, and Challenges. IEEE Commun. Mag. 2017, 55, 80-87. [CrossRef]

96. Chen, T.; Matinmikko, M.; Chen, X.; Zhou, X.; Ahokangas, P. Software defined mobile networks: Concept, survey, and research directions. IEEE Commun. Mag. 2015, 53, 126-133. [CrossRef]

97. Han, B.; Gopalakrishnan, V.; Ji, L.; Lee, S. Network function virtualization: Challenges and opportunities for innovations. IEEE Commun. Mag. 2015, 53, 90-97. [CrossRef]

98. Chen, W.; Liu, C. High-performance user plane function (UPF) for the next generation core networks. IET Netw. 2020, 9, 284-289. [CrossRef]

99. Peters, S.; Khan, M.A. Anticipatory User Plane Management for 5G. In Proceedings of the IEEE 8th International Symposium on Cloud and Service Computing (SC2), Paris, France, 18-21 November 2018.

100. Gärdborn, P. Is QUIC a Better Choice than TCP in the 5G Core Network Service Based Architecture? Master's Thesis, School of Electrical Engineering and Computer Science, KTH Royal Institute of Technology, Stockholm, Sweden, November 2020. Available online: https:// www.diva-portal.org/smash/get/diva2:1520258/FULLTEXT01.pdf (accessed on 31 April 2021).

101. Giordani, M.; Polese, M.; Roy, A.; Castor, D.; Zorzi, M. A Tutorial on Beam Management for 3GPP NR at mmWave Frequencies. IEEE Commun. Surv. Tutor. 2019, 21, 173-196. [CrossRef]

102. Giordani, M.; Mezzavilla, M.; Zorzi, M. Initial Access in 5G mmWave Cellular Networks. IEEE Commun. Mag. 2016, 54, 40-47. [CrossRef]

103. Giordani, M.; Zorzi, M. Improved user tracking in 5G millimeter wave mobile networks via refinement operations. In Proceedings of the 2017 16th Annual Mediterranean Ad Hoc Networking Workshop (Med-Hoc-Net), Budva, Montenegro, 28-30 June 2017.

104. Na, W.; Bae, B.; Cho, S.; Kim, N. DL-TCP: Deep Learning-Based Transmission Control Protocol for Disaster 5G mmWave Networks. IEEE Access 2019, 7, 145134-145144. [CrossRef]

105. Lorincz, J.; Chiaraviglio, L.; Cuomo, F. A measurement study of short-time cell outages in mobile cellular networks. Comput. Commun. 2016, 79, 92-102. [CrossRef]

106. Shahsavari, S.; Khojastepour, M.A.A.; Erkip, E. Robust Beam Tracking and Data Communication in Millimeter Wave Mobile Networks. In Proceedings of the 2019 International Symposium on Modeling and Optimization in Mobile, Ad Hoc, and Wireless Networks (WiOPT), Avignon, France, 3-7 June 2019.

107. Hassan, N.; Yau, K.A.; Wu, C. Edge Computing in 5G: A Review. IEEE Access 2019, 7, 127276-127289. [CrossRef]

108. Mach, P.; Becvar, Z. Mobile edge computing: A survey on architecture and computation offloading. IEEE Commun. Surv. Tutor. 2017, 19, 1628-1656. [CrossRef]

109. Parkvall, S.; Dahlman, E.; Furuskar, A.; Frenne, M. NR: The New 5G Radio Access Technology. IEEE Commun. Stand. Mag. 2017, 1, 24-30. [CrossRef]

110. Lorincz, J.; Bogarelli, M.; Capone, A.; Begusic, D. Heuristic approach for optimized energy savings in wireless access networks. In Proceedings of the 18th International Conference on Software, Telecommunications and Computer Networks (SoftCOM 2010), Split, Croatia, 23-25 September 2010.

111. Lorincz, J.; Matijevic, T.; Petrovic, G. On interdependence among transmit and consumed power of macro base station technologies. Comput. Commun. 2014, 50, 10-28. [CrossRef]

112. Zhang, T.; Mao, S. Machine Learning for End-to-End Congestion Control. IEEE Commun. Mag. 2020, 58, 52-57. [CrossRef] 
113. Wei, W.; Gu, H.; Li, B. Congestion Control: A Renaissance with Machine Learning. IEEE Netw. 2021, 1-8. [CrossRef]

114. Winstein, K.; Balakrishnan, H. TCP ex Machina: Computer-Generated Congestion Control. ACM SIGCOMM Comput. Commun. Rev. 2013, 43, 123-134. [CrossRef]

115. Yan, F.Y.; Ma, J.; Hill, G.D.; Raghavan, D.; Wahby, R.S.; Levis, P.; Winstein, K. Pantheon: The Training Ground for Internet Congestion-control Research. In Proceedings of the USENIX Annual Technical Conference, Berkeley, CA, USA, 11-13 July 2018.

116. Dong, M.; Li, Q.; Zarchy, D.; Godfrey, P.B.; Schapira, M. PCC: Re-architecting congestion control for consistent high performance. In Proceedings of the 12th USENIX Conference on Networked Systems Design and Implementation (NSDI'15), Oakland, CA, USA, 4-6 May 2021.

117. Dong, M.; Meng, T.; Zarchy, D.; Arslan, E.; Gilad, Y.; Godfrey, P.B.; Schapira, M. PCC Vivace: Online-Learning Congestion Control. In Proceedings of the 15th USENIX Conference on Networked Systems Design and Implementation (NSDI'18), Renton, WA, USA, 9-11 April 2018.

118. Jay, N.; Rotman, N.; Godfrey, B.; Schapira, M.; Tamar, A. A Deep Reinforcement Learning Perspective on Internet Congestion Control. In Proceedings of the 36th International Conference on Machine Learning, Long Beach, CA, USA, 9-15 June 2019.

119. Emara, S.; Li, B.; Chen, Y. Eagle: Refining Congestion Control by Learning from the Experts. In Proceedings of the IEEE INFOCOM 2020-IEEE Conference on Computer. Communications, Toronto, ON, Canada, 6-9 July 2020.

120. Abbasloo, S.; Yen, C.-Y.; Chao, H.J. Classic Meets Modern: A Pragmatic Learning-Based Congestion Control for the Internet. In Proceedings of the Annual Conference of the ACM Special Interest Group on Data Communication on the Applications, Technologies, Architectures, and Protocols for Computer Communication (SIGCOMM '20), New York, NY, USA, 10-14 August 2020.

121. Feng, M.; Mao, S. Dealing with Limited Backhaul Capacity in Millimeter-Wave Systems: A Deep Reinforcement Learning Approach. IEEE Commun. Mag. 2019, 57, 50-55. [CrossRef]

122. Kong, Y.; Zang, H.; Ma, X. Improving TCP Congestion Control with Machine Intelligence. In Proceedings of the 2018 Workshop on Network Meets AI \& ML, New York, NY, USA, 24 August 2018.

123. Li, W.; Zhou, F.; Chowdhury, K.R.; Meleis, W. QTCP: Adaptive Congestion Control with Reinforcement Learning. IEEE Trans. Netw. Sci. Eng. 2019, 6, 445-458. [CrossRef]

124. Najm, I.A.; Khalaf, A.; Lloret, J.; Bosch, I. Machine Learning Prediction Approach to Enhance Congestion Control in 5G IoT Environment. Electronics 2019, 8, 607. [CrossRef] 